MATHEMATICS OF COMPUTATION

Volume 72, Number 242, Pages 729-756

S 0025-5718(02)01431-X

Article electronically published on March 8, 2002

\title{
NYSTRÖM-CLENSHAW-CURTIS QUADRATURE FOR INTEGRAL EQUATIONS WITH DISCONTINUOUS KERNELS
}

\author{
SHEON-YOUNG KANG, ISRAEL KOLTRACHT, AND GEORGE RAWITSCHER
}

AbStRaCt. A new highly accurate numerical approximation scheme based on a Gauss type Clenshaw-Curtis quadrature for Fredholm integral equations of the second kind

$$
x(t)+\int_{a}^{b} k(t, s) x(s) d s=y(t),
$$

whose kernel $k(t, s)$ is either discontinuous or not smooth along the main diagonal, is presented. This scheme is of spectral accuracy when $k(t, s)$ is infinitely differentiable away from the diagonal $t=s$. Relation to the singular value decomposition is indicated. Application to integro-differential Schrödinger equations with nonlocal potentials is given.

\section{InTRODUCTION}

Let the integral operator,

$$
(K x)(t)=\int_{a}^{b} k(t, s) x(s) d s, \quad a \leq t \leq b,
$$

map $C_{[a, b]}^{q}, q>1$, into itself. In the present paper, we consider the numerical solution of the corresponding Fredholm integral equation of the second kind,

$$
x(t)+\int_{a}^{b} k(t, s) x(s) d s=y(t), \quad y \in C^{q}, \quad a \leq t \leq b .
$$

When the kernel $k(t, s)$ has a discontinuity either by itself or in its partial derivatives along the main diagonal $t=s$, one cannot expect a high accuracy Nyström quadrature based on Newton-Cotes or Gaussian integration rules, see, e.g., Figure 2 of Section 5, since, except for the trapezium rule, the standard error bounds for these rules are not applicable. If the function $x(t)$ were known, then the discretization of the integral operator in (11) would be straightforward: for any fixed $t$ the interval $[a, b]$ can be partitioned so that in each subinterval the integrand is smooth. When $x(t)$ is unknown it is generally not possible to get an accurate discretization of (1) with $x(t)$ and $x(s)$ sampled at the same support points, without using some

Received by the editor March 29, 2001 and, in revised form, July 9, 2001.

2000 Mathematics Subject Classification. Primary 45B05, 45J05, 65Rxx, 65R20, 81U10.

Key words and phrases. Discontinuous kernels, fast algorithms, nonlocal potentials.

The work of the first author is partially supported by a fellowship from alumni of Mathematics Department, Chungnam National University, Korea. 
sort of interpolation. The main purpose of this paper is to introduce a high accuracy Nyström-Gauss quadrature for a certain class of discontinuous kernels which we call semi-smooth, for which this difficulty can be overcome.

Definition 1. A kernel $k(t, s)$ is called p-semi-smooth, if

$$
k(t, s)= \begin{cases}k_{1}(t, s) & \text { if } a \leq s \leq t \\ k_{2}(t, s) & \text { if } t \leq s \leq b,\end{cases}
$$

where $k_{1,2}(t, s) \in C_{[a, b] \times[a, b]}^{p}$ for some $p>1$.

The Gauss type integration rule which we use here is the Clenshaw-Curtis rule, 7], and hence the resulting quadrature is called Nyström-Clenshaw-Curtis, or NCC for short.

Note that for our purpose each of the auxiliary kernels $k_{1}(t, s)$ and $k_{2}(t, s)$ must be defined in the whole square $[a, b] \times[a, b]$. The convergence of our method is of or$\operatorname{der} O\left(n^{1-r}\right)$, where $r=\min \{p, q\}$. When $r=\infty$, the convergence is superalgebraic, or spectral. For kernels with some singularities, when the obtained error estimates are not applicable, the method still shows good accuracy on numerical examples. The 2-step method of deferred approach to the limit, based on the trapezium rule (see, e.g., [5], p 363), works well for nonsingular kernels, but it is much more time consuming, for comparable accuracy, and is not applicable to kernels with singularities. For kernels with singularities of certain types, corrected trapezoidal rules of [29], [17] and [2] can be used.

A well-known example of semi-smooth kernels are the semi-separable kernels, with $k_{1}$ and $k_{2}$ being of a low rank $\alpha, k_{i}(t, s)=\sum_{j=1}^{\alpha} f_{j}^{(i)}(t) g_{j}^{(i)}(s), \quad i=1,2$. In fact, semi-smooth kernels can be approximated with semi-separable kernels using the singular value decomposition of $k_{1,2}(t, s)$. However, NCC is less costly and more accurate than the discretization based on the semi-separable approximation. The classical Green kernels for two point boundary value problems are semi-separable of rank $\alpha=2$. Semi-separable kernels of rank $\alpha=4$ occur in nuclear scattering with exchange terms, [23]. For low rank semi-separable kernels, the numerical techniques developed in [15] and [13] are adequate and give fast and accurate solutions. Semi-smooth kernels which are not semi-separable occur in nuclear scattering with nonlocal potentials which are not of the exchange type, as described in the Appendix. For such kernels the discretization technique developed in the present paper is appropriate. This situation is examined in more detail in Section 6 .

Another useful example of semi-smooth kernels is the displacement kernels, $k(t, s)=k(|t-s|)$. Such kernels occur in radiative transfer, Wiener filter theory, resonance scattering, etc. We refer to [16] for an up-to-date review of the literature on Wiener-Hopf type integral equations with displacement kernels. Our discretization technique is highly accurate for such kernels, and at the same time preserves the displacement structure in the discrete equations.

In Section 2, we describe the discretization of equation (11) based on the NyströmClenshaw-Curtis quadrature for a smooth kernel $k(t, s)$. This discretization is different from the usual Gauss-Chebyshev quadrature (e.g., Delves-Mohamed, [8]) and from that of Reichel [25], based on Chebyshev polynomial expansions. In Section 3 we consider semi-smooth kernels and show that the application of NyströmClenshaw-Curtis quadrature results in a linear system of equations whose coefficient matrix is defined in terms of Schur, or componentwise, products of given matrices. The accuracy of approximation is determined by the smoothness of $k_{1}$ and $k_{2}$ only, 
and is not affected by the discontinuity along the diagonal $t=s$. For smooth kernels this discretization is identical with the one described in Section 2. In Section 4 we detail the relation of NCC to the singular value decomposition of $k_{1,2}(t, s)$. In Section 5 we describe the NCC composite rule. In Section 6 we describe numerical experiments and comparisons with some existing methods for kernels with various discontinuities and singularities. In Section 7 we apply our technique to the solution of radial Schrödinger integro-differential equations with a nonlocal potential. The physical motivation for such integro-differential equations is given in the Appendix.

\section{Discretization of a SMOOTh KeRnel}

Let $k(t, s)$ be differentiable in $t$ and $s$. Assume that for any $a \leq t_{k} \leq b$, $k\left(t_{k}, s\right) x(s)$ as a function of $s$ can be expanded in a finite set of polynomials, i.e.,

$$
k\left(t_{k}, s\right) x(s)=\sum_{j=0}^{n} \alpha_{k j} T_{j}(s), \quad-1 \leq s \leq 1,
$$

where $T_{j}(s)=\cos (j \arccos (s)), \quad j=0,1, \ldots, n$, are the Chebyshev polynomials. Without any loss of generality we assume for now that $a=-1$ and $b=1$ in equation (11). Let

$$
F(r)=\int_{-1}^{r} k\left(t_{k}, s\right) x(s) d s=\sum_{j=0}^{n+1} \beta_{k j} T_{j}(r) .
$$

Clenshaw and Curtis [7] showed that

$$
\left[\beta_{k 0}, \beta_{k 1}, \ldots, \beta_{k n+1}\right]^{T}=\mathbf{S}_{L}\left[\alpha_{k 0}, \alpha_{k 1}, \ldots, \alpha_{k n}\right]^{T},
$$

where

$$
\mathbf{S}_{L}=\left[\begin{array}{llllll}
1 & 1 & -1 & 1 & \cdots & (-1)^{n} \\
0 & 1 & 0 & 0 & \cdots & 0 \\
0 & 0 & 1 & 0 & \cdots & 0 \\
\vdots & \vdots & \vdots & \ddots & \ddots & \vdots \\
0 & 0 & 0 & 0 & 1 & 0 \\
0 & 0 & \cdots & 0 & 0 & 1
\end{array}\right]\left[\begin{array}{llllll}
0 & 0 & 0 & 0 & \cdots & 0 \\
1 & 0 & \frac{-1}{2} & 0 & \cdots & 0 \\
0 & \frac{1}{4} & 0 & \frac{-1}{4} & \cdots & 0 \\
\vdots & \vdots & \ddots & \ddots & \ddots & \vdots \\
0 & \cdots & 0 & \frac{1}{2(n-1)} & 0 & \frac{-1}{2(n-1)} \\
0 & \cdots & 0 & 0 & \frac{1}{2 n} & 0
\end{array}\right]
$$

is the so-called left spectral integration matrix. Here $[\nu]^{T}$ denotes the transpose of the column vector $\nu$. Since $T_{j}(1)=1$ for $j=0,1, \ldots, n$, it follows that

$$
\begin{aligned}
F(1) & =\int_{-1}^{1} k\left(t_{k}, s\right) x(s) d s=\sum_{j=0}^{n+1} \beta_{k j} \\
& =[1, \ldots, 1]\left[\beta_{k 0}, \beta_{k 1}, \ldots, \beta_{k n+1}\right]^{T}=[1, \ldots, 1] \mathbf{S}_{L}\left[\alpha_{k 0}, \alpha_{k 1}, \ldots, \alpha_{k n}\right]^{T} .
\end{aligned}
$$

Let $\tau_{k}, k=0,1, \ldots, n$, denote the zeros of $T_{n+1}$, viz.,

$$
\tau_{k}=\cos \frac{(2 k+1) \pi}{2(n+1)}
$$

so that

$$
T_{j}\left(\tau_{k}\right)=\cos \frac{(2 k+1) j \pi}{2(n+1)}, \quad k, j=0,1, \ldots, n .
$$


Substituting $s=\tau_{k}, k=0,1, \ldots, n$, into (2), we obtain that

$$
\left[\begin{array}{c}
\alpha_{k 0} \\
\alpha_{k 1} \\
\vdots \\
\alpha_{k n}
\end{array}\right]=\mathbf{C}^{-1}\left[\begin{array}{c}
k\left(t_{k}, \tau_{0}\right) x\left(\tau_{0}\right) \\
k\left(t_{k}, \tau_{1}\right) x\left(\tau_{1}\right) \\
\vdots \\
k\left(t_{k}, \tau_{n}\right) x\left(\tau_{n}\right)
\end{array}\right]
$$

where $\mathbf{C}^{-1}$ is an inverse of the discrete cosine transformation matrix $\mathbf{C}$ whose elements are specified by

$$
\mathbf{C}_{k j}=T_{j}\left(\tau_{k}\right), \quad k, j=0,1, \ldots, n .
$$

The matrix $\mathbf{C}$ has orthogonal columns, that is, $\mathbf{C}^{T} \mathbf{C}=\operatorname{diag}\left(n, \frac{n}{2}, \ldots, \frac{n}{2}\right)$. Therefore, $\mathbf{C}^{-1}=\operatorname{diag}\left(\frac{1}{n}, \frac{2}{n}, \ldots, \frac{2}{n}\right) \mathbf{C}^{T}$. By choosing $t_{k}$ in (2) to be Chebyshev points and by substituting $t=\tau_{k}$ into (1), we get

$y\left(\tau_{k}\right)=x\left(\tau_{k}\right)+[1, \ldots, 1] \mathbf{S}_{L} \mathbf{C}^{-1} \operatorname{diag}\left(k\left(\tau_{k}, \tau_{0}\right), k\left(\tau_{k}, \tau_{1}\right), \ldots, k\left(\tau_{k}, \tau_{n}\right)\right)\left[\begin{array}{c}x\left(\tau_{0}\right) \\ x\left(\tau_{1}\right) \\ \vdots \\ x\left(\tau_{n}\right)\end{array}\right]$.

Introducing $\left[\sigma_{0}, \sigma_{1}, \ldots, \sigma_{n}\right]=[1,1, \ldots, 1] \mathbf{S}_{L} \mathbf{C}^{-1}$, we can write

$$
y\left(\tau_{k}\right)=\left[\sigma_{0}, \sigma_{1}, \ldots, \sigma_{n}\right] \operatorname{diag}\left(k\left(\tau_{k}, \tau_{0}\right), k\left(\tau_{k}, \tau_{1}\right), \ldots, k\left(\tau_{k}, \tau_{n}\right)\right)\left[\begin{array}{c}
x\left(\tau_{0}\right) \\
x\left(\tau_{1}\right) \\
\vdots \\
x\left(\tau_{n}\right)
\end{array}\right],
$$

or equivalently,

$$
y\left(\tau_{k}\right)=\left[k\left(\tau_{k}, \tau_{0}\right), k\left(\tau_{k}, \tau_{1}\right), \ldots, k\left(\tau_{k}, \tau_{n}\right)\right] \operatorname{diag}\left(\sigma_{0}, \sigma_{1}, \ldots, \sigma_{n}\right)\left[\begin{array}{c}
x\left(\tau_{0}\right) \\
x\left(\tau_{1}\right) \\
\vdots \\
x\left(\tau_{n}\right)
\end{array}\right] .
$$

Therefore the discretization of the equation (1) for the case $a=-1$ and $b=1$ is as follows:

$$
\left[\mathbf{I}+\mathbf{K D}_{\sigma}\right] \overline{\mathbf{x}}=\overline{\mathbf{y}}
$$

where

$$
\begin{aligned}
\mathbf{K} & =\left(k\left(\tau_{i}, \tau_{j}\right)\right)_{i, j=0}^{n}, \\
\mathbf{D}_{\sigma} & =\operatorname{diag}\left(\sigma_{0}, \sigma_{1}, \ldots, \sigma_{n}\right), \\
\overline{\mathbf{x}} & =\left[x\left(\tau_{0}\right), x\left(\tau_{1}\right), \ldots, x\left(\tau_{n}\right)\right]^{T}, \\
\overline{\mathbf{y}} & =\left[y\left(\tau_{0}\right), y\left(\tau_{1}\right), \ldots, y\left(\tau_{n}\right)\right]^{T} .
\end{aligned}
$$

The formulas (3) can be generalized for intervals $[a, b]$ other than $[-1,1]$ by the linear change of the variable $h(\tau)=\frac{1}{2}(b-a) \tau+\frac{1}{2}(a+b)$. Thus if $\eta_{j}=h\left(\tau_{j}\right), \quad j=$ $0,1, \ldots, n$, we have

$$
\left[\mathbf{I}+\frac{b-a}{2} \mathbf{K D}_{\sigma}\right] \overline{\mathbf{x}}=\overline{\mathbf{y}}
$$


where

$$
\begin{aligned}
\mathbf{K} & =\left(k\left(\eta_{i}, \eta_{j}\right)\right)_{i, j=0}^{n}, \quad \mathbf{D}_{\sigma}=\operatorname{diag}\left(\sigma_{0}, \sigma_{1}, \ldots, \sigma_{n}\right), \\
\overline{\mathbf{x}} & =\left[x\left(\eta_{0}\right), x\left(\eta_{1}\right), \ldots, x\left(\eta_{n}\right)\right]^{T}, \quad \overline{\mathbf{y}}=\left[y\left(\eta_{0}\right), y\left(\eta_{1}\right), \ldots, y\left(\eta_{n}\right)\right]^{T} .
\end{aligned}
$$

The accuracy of this discretization when $k$ and $x$ are not polynomials is discussed in a more general setting in the next section.

\section{Gauss type quadrature For a SEMi-Smooth Kernel}

We consider now more general semi-smooth kernels, as in Definition 1, for which we write

$$
x(t)+\int_{a}^{t} k_{1}(t, s) x(s) d s+\int_{t}^{b} k_{2}(t, s) x(s) d s=y(t), \quad a \leq t \leq b .
$$

In this section we describe the numerical technique for discretizing the equation (4). It is based on the Clenshaw-Curtis quadrature described in Section 2, which is well suited for computing antiderivatives. First assume that $a=-1, b=1$ and let

$$
F(t)=\int_{-1}^{t} k_{1}(t, s) x(s) d s, \quad G(t, \lambda)=\int_{-1}^{\lambda} k_{1}(t, s) x(s) d s,
$$

such that $F(t)=G(t, t)$, and let

$$
H(t)=\int_{t}^{1} k_{2}(t, s) x(s) d s, \quad J(t, \lambda)=\int_{\lambda}^{1} k_{2}(t, s) x(s) d s .
$$

Further, assume that $k_{1}\left(t_{k}, s\right) x(s)$ can be expanded in a finite set of polynomials, i.e., $k_{1}\left(t_{k}, s\right) x(s)=\sum_{i=0}^{n} \alpha_{k i} T_{i}(s)$. As we have seen in Section 2, if

$$
G\left(t_{k}, \lambda\right)=\sum_{j=0}^{n+1} \beta_{k j} T_{j}(\lambda)
$$

then

$$
\left[\beta_{k 0}, \beta_{k 1}, \ldots, \beta_{k n+1}\right]^{T}=\mathbf{S}_{L}\left[\alpha_{k 0}, \alpha_{k 1}, \ldots, \alpha_{k n}\right]^{T} .
$$

Similarly, assume that $k_{2}\left(t_{k}, s\right) x(s)=\sum_{j=0}^{n} \tilde{\alpha}_{k j} T_{j}(s)$. If

$$
J\left(t_{k}, \lambda\right)=\int_{\lambda}^{1} k_{2}\left(t_{k}, s\right) x(s) d s=\sum_{j=0}^{n+1} \tilde{\beta}_{k j} T_{j}(\lambda),
$$

then

$$
\left[\tilde{\beta}_{k 0}, \tilde{\beta}_{k 1}, \ldots, \tilde{\beta}_{k n+1}\right]^{T}=\mathbf{S}_{R}\left[\tilde{\alpha}_{k 0}, \tilde{\alpha}_{k 1}, \ldots, \tilde{\alpha}_{k n}\right]^{T}
$$

where

$$
\mathbf{S}_{R}=\left[\begin{array}{llllll}
1 & 1 & 1 & 1 & \cdots & 1 \\
0 & -1 & 0 & 0 & \cdots & 0 \\
0 & 0 & -1 & 0 & \cdots & 0 \\
\vdots & \vdots & \vdots & \ddots & \ddots & \vdots \\
0 & 0 & 0 & 0 & -1 & 0 \\
0 & 0 & \cdots & 0 & 0 & -1
\end{array}\right]\left[\begin{array}{llllll}
0 & 0 & 0 & 0 & \cdots & 0 \\
1 & 0 & \frac{-1}{2} & 0 & \cdots & 0 \\
0 & \frac{1}{4} & 0 & \frac{-1}{4} & \cdots & 0 \\
\vdots & \vdots & \ddots & \ddots & \ddots & \vdots \\
0 & \cdots & 0 & \frac{1}{2(n-1)} & 0 & \frac{-1}{2(n-1)} \\
0 & \cdots & 0 & 0 & \frac{1}{2 n} & 0
\end{array}\right]
$$


is the right spectral integration matrix. Let $\tau_{k}, k=0,1, \ldots, n$, denote the zeros of $T_{n+1}$. Substituting $\lambda=\tau_{k}, k=0,1, \ldots, n$, into (5), we obtain that

$$
\left[\begin{array}{c}
G\left(t_{k}, \tau_{0}\right) \\
G\left(t_{k}, \tau_{1}\right) \\
\vdots \\
G\left(t_{k}, \tau_{n}\right)
\end{array}\right]=\mathbf{C S}_{L} \mathbf{C}^{-1} \operatorname{diag}\left(k_{1}\left(t_{k}, \tau_{0}\right), \ldots, k_{1}\left(t_{k}, \tau_{n}\right)\right)\left[\begin{array}{c}
x\left(\tau_{0}\right) \\
\vdots \\
x\left(\tau_{n}\right)
\end{array}\right]
$$

and, similarly,

$$
\left[\begin{array}{c}
J\left(t_{k}, \tau_{0}\right) \\
J\left(t_{k}, \tau_{1}\right) \\
\vdots \\
J\left(t_{k}, \tau_{n}\right)
\end{array}\right]=\mathbf{C S}_{R} \mathbf{C}^{-1} \operatorname{diag}\left(k_{2}\left(t_{k}, \tau_{0}\right), \ldots, k_{2}\left(t_{k}, \tau_{n}\right)\right)\left[\begin{array}{c}
x\left(\tau_{0}\right) \\
\vdots \\
x\left(\tau_{n}\right)
\end{array}\right] .
$$

We remark that in writing the equality sign in (6) and (7), we assume that $\beta_{n+1}$ is set to zero. This is an acceptable assumption, because in practical applications the kernel $k(t, s)$ and the right-hand side $y(t)$ are not polynomials and the equality in (2) is only approximate. In fact, following Clenshaw and Curtis, [7], we use the size of the $\alpha_{n}$ 's and $\beta_{n}$ 's as a readily available tool to control the accuracy of approximation, and chose $n$ large enough so that the $\alpha_{n}$ 's and $\beta_{n}$ 's are less than a prescribed tolerance (see also Remark 1 in Section 5). Therefore setting $\beta_{n+1}$ to zero does not affect the overall accuracy.

Since $F\left(\tau_{k}\right)=G\left(\tau_{k}, \tau_{k}\right)$, we get

$$
\begin{aligned}
F\left(\tau_{k}\right) & =[0, \ldots, 0,1,0, \ldots, 0] \mathbf{C S}_{L} \mathbf{C}^{-1} \operatorname{diag}\left(k_{1}\left(\tau_{k}, \tau_{0}\right), \ldots, k_{1}\left(\tau_{k}, \tau_{n}\right)\right)\left[\begin{array}{c}
x\left(\tau_{0}\right) \\
\vdots \\
x\left(\tau_{n}\right)
\end{array}\right] \\
& =\left[w_{k 0}, w_{k 1}, \ldots, w_{k n}\right] \operatorname{diag}\left(k_{1}\left(\tau_{k}, \tau_{0}\right), \ldots, k_{1}\left(\tau_{k}, \tau_{n}\right)\right)\left[\begin{array}{c}
x\left(\tau_{0}\right) \\
\vdots \\
x\left(\tau_{n}\right)
\end{array}\right] \\
& =\left[w_{k 0}, w_{k 1}, \ldots, w_{k n}\right] \operatorname{diag}\left(x\left(\tau_{0}\right), \ldots, x\left(\tau_{n}\right)\right)\left[\begin{array}{c}
k_{1}\left(\tau_{k}, \tau_{0}\right) \\
\vdots \\
k_{1}\left(\tau_{k}, \tau_{n}\right)
\end{array}\right]
\end{aligned}
$$

where $\left[w_{k 0}, \ldots, w_{k n}\right]$ is the $(k+1)$-st row of the matrix $\mathbf{W} \stackrel{\text { def }}{=} \mathbf{C S}_{L} \mathbf{C}^{-1}$. We need now the following identity, which can be verified by direct calculation.

Lemma 1. Let $\mathbf{A}$ and $\mathbf{B}$ be $n \times n$ matrices and $\mathbf{c}=\left[c_{1}, \ldots, c_{n}\right]^{T}$. Then $(\mathbf{A} \circ \mathbf{B}) \mathbf{c}=$ $\operatorname{diag}\left(\mathbf{A} \operatorname{diag}\left(c_{1}, \ldots, c_{n}\right) \mathbf{B}^{T}\right)$, where $\mathbf{A} \circ \mathbf{B}$ denotes the Schur product of $\mathbf{A}$ and $\mathbf{B}$, $(\mathbf{A} \circ \mathbf{B})_{i j}=a_{i j} b_{i j}, \quad i, j=1, \ldots, n$.

Using this lemma, we find that

$$
\left[\begin{array}{c}
F\left(\tau_{0}\right) \\
F\left(\tau_{1}\right) \\
\vdots \\
F\left(\tau_{n}\right)
\end{array}\right]=\operatorname{diag}\left(\mathbf{W} \operatorname{diag}\left(x\left(\tau_{0}\right), \ldots, x\left(\tau_{n}\right)\right) \mathbf{K}_{1}^{T}\right)=\left(\mathbf{W} \circ \mathbf{K}_{1}\right)\left[\begin{array}{c}
x\left(\tau_{0}\right) \\
\vdots \\
x\left(\tau_{n}\right)
\end{array}\right]
$$


where $\mathbf{K}_{1}=\left(k_{1}\left(\tau_{i}, \tau_{j}\right)\right)_{i, j=0}^{n}$. Similarly,

$$
\left[\begin{array}{c}
H\left(\tau_{0}\right) \\
H\left(\tau_{1}\right) \\
\vdots \\
H\left(\tau_{n}\right)
\end{array}\right]=\left(\mathbf{V} \circ \mathbf{K}_{2}\right)\left[\begin{array}{c}
x\left(\tau_{0}\right) \\
\vdots \\
x\left(\tau_{n}\right)
\end{array}\right],
$$

where $\mathbf{V}=\mathbf{C S}_{R} \mathbf{C}^{-1}$. The formulas (8) and (9) can be generalized for an interval $[a, b]$ other than $[-1,1]$ by the linear change of variables $h(\tau)=\frac{1}{2}(b-a) \tau+\frac{1}{2}(a+b)$. Thus if $\eta_{j}=h\left(\tau_{j}\right), \quad j=0,1, \ldots, n$, and with the notation

$$
F_{a}(t)=\int_{a}^{t} k_{1}(t, s) x(s) d s, \quad H_{b}(t)=\int_{t}^{b} k_{2}(t, s) x(s) d s,
$$

we have

$$
\left[\begin{array}{c}
F_{a}\left(\eta_{0}\right) \\
F_{a}\left(\eta_{1}\right) \\
\vdots \\
F_{a}\left(\eta_{n}\right)
\end{array}\right]=\frac{b-a}{2}\left(\mathbf{W} \circ \mathbf{K}_{1}\right)\left[\begin{array}{c}
x\left(\eta_{0}\right) \\
x\left(\eta_{1}\right) \\
\vdots \\
x\left(\eta_{n}\right)
\end{array}\right]
$$

and

$$
\left[\begin{array}{c}
H_{b}\left(\eta_{0}\right) \\
H_{b}\left(\eta_{1}\right) \\
\vdots \\
H_{b}\left(\eta_{n}\right)
\end{array}\right]=\frac{b-a}{2}\left(\mathbf{V} \circ \mathbf{K}_{2}\right)\left[\begin{array}{c}
x\left(\eta_{0}\right) \\
x\left(\eta_{1}\right) \\
\vdots \\
x\left(\eta_{n}\right)
\end{array}\right]
$$

Using (10) and (11), we can now discretize the equation (4) as follows:

$$
\left[\mathbf{I}+\frac{b-a}{2}\left(\mathbf{W} \circ \mathbf{K}_{1}+\mathbf{V} \circ \mathbf{K}_{2}\right)\right] \overline{\mathbf{x}}=\overline{\mathbf{y}},
$$

where $\overline{\mathbf{x}}=\left[x\left(\eta_{0}\right), \ldots, x\left(\eta_{n}\right)\right]^{T}$ and $\overline{\mathbf{y}}=\left[y\left(\eta_{0}\right), \ldots, y\left(\eta_{n}\right)\right]^{T}$. We refer to this discretization as the NCC quadrature. Next we show that if the kernel function $k(t, s)$ is smooth, such that $k_{1}=k_{2}$, then the discretization (12) reduces to (3).

Proposition 1. Suppose that $k(t, s) \in C_{[a, b] \times[a, b]}^{p}$, and that $k_{1}(t, s)=k_{2}(t, s)=$ $k(t, s)$. Then,

$$
\left[\mathbf{I}+\frac{b-a}{2}\left(\mathbf{W} \circ \mathbf{K}_{1}+\mathbf{V} \circ \mathbf{K}_{2}\right)\right] \overline{\mathbf{x}}=\left[\mathbf{I}+\frac{b-a}{2} \mathbf{K D}_{\sigma}\right] \overline{\mathbf{x}} .
$$

Proof. Without any loss of generality we assume that $a=-1$ and $b=1$. For $t=t_{k},-1 \leq t_{k} \leq 1$, we see that the equality

$$
x\left(t_{k}\right)+G\left(t_{k}, \lambda\right)+J\left(t_{k}, \lambda\right)=y\left(t_{k}\right)
$$


holds for any $\lambda,-1 \leq \lambda \leq 1$. Therefore if $\lambda=1$, then $J\left(t_{k}, 1\right)=0$ and

$$
\begin{aligned}
G\left(t_{k}, 1\right) & =\sum_{j=0}^{n} \beta_{k j}=[1, \ldots, 1] \mathbf{S}_{L}\left[\begin{array}{c}
\alpha_{k 0} \\
\vdots \\
\alpha_{k n}
\end{array}\right] \\
& =[1, \ldots, 1] \mathbf{S}_{L} \mathbf{C}^{-1} \operatorname{diag}\left(k\left(t_{k}, \tau_{0}\right), \ldots, k\left(t_{k}, \tau_{n}\right)\right)\left[\begin{array}{c}
x\left(\tau_{0}\right) \\
\vdots \\
x\left(\tau_{n}\right)
\end{array}\right] \\
& =\left[\sigma_{0}, \sigma_{1}, \ldots, \sigma_{n}\right] \operatorname{diag}\left(k\left(t_{k}, \tau_{0}\right), \ldots, k\left(t_{k}, \tau_{n}\right)\right)\left[\begin{array}{c}
x\left(\tau_{0}\right) \\
\vdots \\
x\left(\tau_{n}\right)
\end{array}\right] \\
& {\left[k\left(t_{k}, \tau_{0}\right), k\left(t_{k}, \tau_{1}\right), \ldots, k\left(t_{k}, \tau_{n}\right)\right] \operatorname{diag}\left(\sigma_{0}, \sigma_{1}, \ldots, \sigma_{n}\right)\left[\begin{array}{c}
x\left(\tau_{0}\right) \\
x\left(\tau_{1}\right) \\
\vdots \\
x\left(\tau_{n}\right)
\end{array}\right] . }
\end{aligned}
$$

Substituting $t_{k}=\tau_{k}$ for $k=0,1, \ldots, n$ into (13), we obtain that

$$
\left[\mathbf{I}+\mathbf{K D}_{\sigma}\right] \overline{\mathbf{x}}=\overline{\mathbf{y}} \text {. }
$$

The assertion now follows.

We compared the numerical behavior of the discretization (31) and (12) for a number of smooth kernels and found that numerical answers differed in accuracy at the level of machine precision only.

We now estimate the accuracy of approximation of the integral equation (4) with the linear system of equations (12). The following property of Chebyshev expansions can be derived along the lines of an argument in Gottlieb and Orszag ([14], p.29).

Proposition 2. Let $f \in C^{r}[-1,1], \quad r>1, \quad$ and let

$$
f(t)=\sum_{j=0}^{\infty} \alpha_{j} T_{j}(t), \quad-1 \leq t \leq 1
$$

Then

$$
\left|\alpha_{j}\right| \leq \frac{2}{\pi} \int_{0}^{\pi}\left|\frac{d^{r}}{d \theta^{r}} f(\cos \theta)\right| d \theta \frac{1}{j^{r}}=\frac{c}{j^{r}}
$$

and

$$
\left|f(t)-\sum_{j=0}^{n} \alpha_{j} T_{j}(t)\right| \leq \frac{c}{r-1} \frac{1}{n^{r-1}} .
$$

This implies that if $f(r)$ is analytic then the convergence of Chebyshev expansions is superalgebraic. Now let $F_{l}(x)=\int_{-1}^{x} f(t) d t$ and $F_{r}(x)=\int_{x}^{1} f(t) d t$. The following result can be found in Greengard and Rokhlin [15].

Proposition 3. Suppose that $f \in C_{[-1,1]}^{r}, \quad r>1$, and that $\bar{f}=\left(f\left(\tau_{0}\right), \ldots, f\left(\tau_{n}\right)\right)^{T}$ is the vector of the function values at the roots of $T_{n+1}(x)$. Suppose further that $\bar{F}_{l}$ 
and $\bar{F}_{r}$ are defined by

$$
\bar{F}_{l}=\left(F_{l}\left(\tau_{0}\right), \ldots, F_{l}\left(\tau_{n}\right)\right)^{T}, \quad \bar{F}_{r}=\left(F_{r}\left(\tau_{0}\right), \ldots, F_{r}\left(\tau_{n}\right)\right)^{T}
$$

Then

$$
\left\|\bar{F}_{l}-\mathbf{C S}_{L} \mathbf{C}^{-1} \bar{f}\right\|_{\infty}=O\left(\frac{1}{n^{r-1}}\right)
$$

and

$$
\left\|\bar{F}_{r}-\mathbf{C S}_{R} \mathbf{C}^{-1} \bar{f}\right\|_{\infty}=O\left(\frac{1}{n^{r-1}}\right) .
$$

Furthermore, all elements of the matrices $\mathbf{C S}_{L} \mathbf{C}^{-1}$ and $\mathbf{C S}_{R} \mathbf{C}^{-1}$ are strictly positive.

Now let $\eta_{i}=\frac{b-a}{2} \tau_{i}+\frac{a+b}{2}$, where $\tau_{i}$ is a zero of $T_{n+1}(x)$, for $i=0,1, \ldots, n$, be the shifted Chebyshev points, and let $\hat{\mathbf{x}}=\left(x\left(\eta_{0}\right), x\left(\eta_{1}\right), \ldots, x\left(\eta_{n}\right)\right)^{T}$ be the vector of values of solution $x(t)$ of equation (4) at $\eta_{i}$. The following proposition follows immediately from standard properties of the Riemann integral (see, e.g., [26], p.105).

Proposition 4. Let $k(t, s)$ be p-semi-smooth and let $y(t) \in C_{[a, b]}^{q}$, with $r=$ $\min \{p, q\}>1$. Let the equation (44) define an invertible operator on $C_{[a, b]}^{r}$. Then $x \in C_{[a, b]}^{r}$.

Now let

$$
\bar{F}_{a}=\left(F_{a}\left(\eta_{0}\right), \ldots, F_{a}\left(\eta_{n}\right)\right)^{T}
$$

and

$$
\bar{H}_{b}=\left(H_{b}\left(\eta_{0}\right), \ldots, H_{b}\left(\eta_{n}\right)\right)^{T} .
$$

It follows from Proposition 3 that in the conditions of Proposition 4

$$
\left\|\bar{F}_{a}-\frac{b-a}{2}\left(\mathbf{W} \circ \mathbf{K}_{1}\right) \hat{\mathbf{x}}\right\|_{\infty}=O\left(\frac{1}{n^{r-1}}\right)
$$

and

$$
\left\|\bar{H}_{b}-\frac{b-a}{2}\left(\mathbf{V} \circ \mathbf{K}_{2}\right) \hat{\mathbf{x}}\right\|_{\infty}=O\left(\frac{1}{n^{r-1}}\right) .
$$

Combining the above results, we obtain the following estimate for the residual.

Theorem 1. Let $\overline{\mathbf{x}}$ be a solution vector of the equation (12), and $\hat{\mathbf{x}}$ the vector of values of the solution $x(t)$ at $t=\eta_{i}, i=0,1, \ldots, n$. Suppose that $k(t, s)$ is $p$-semismooth, and that $y(t) \in C_{[a, b]}^{q}$. Suppose further that the equation (4) defines an invertible operator on $C_{[a, b]}^{r}$, where $r=\min \{p, q\}>1$. Then,

$$
\left\|\left(\mathbf{I}+\frac{b-a}{2}\left(\mathbf{W} \circ \mathbf{K}_{1}+\mathbf{V} \circ \mathbf{K}_{2}\right)\right)(\hat{\mathbf{x}}-\overline{\mathbf{x}})\right\|_{\infty}=O\left(\frac{1}{n^{r-1}}\right) .
$$

It follows from the collectively compact operator theory, see Anselone [3], that for sufficiently large $n$ the matrices $\mathbf{I}+\frac{b-a}{2}\left(\mathbf{W} \circ \mathbf{K}_{1}+\mathbf{V} \circ \mathbf{K}_{2}\right)$, which depend on $n$, are invertible and their inverses are uniformly bounded. Therefore Theorem 3.1 implies that for increasing $n$, the convergence of $\bar{x}$ to $\hat{x}$ is of order $O\left(n^{1-r}\right)$. If $p=q=\infty$, then the convergence is superalgebraic. Numerical examples in Section 5 indeed demonstrate this type of convergence. 


\section{SVD AND SEMI-SEPARABLE APPROXIMATION}

The Clenshaw-Curtis quadrature is very effective for semi-separable kernels,

$$
k(t, s)= \begin{cases}\sum_{j=1}^{\alpha} f_{j}^{(i)}(t) g_{j}^{(i)}(s) & \text { if } t>s \\ \sum_{j=1}^{\alpha} p_{j}^{(i)}(t) q_{j}^{(i)}(s) & \text { if } t<s\end{cases}
$$

see [15]. A natural way to extend its application to semi-smooth kernels is by approximating semi-smooth kernels with semi-separable ones. This can be done in the following way. Let

$$
k_{1}(t, s)=\sum_{j=1}^{\infty} \sigma_{j}^{(1)} \phi_{j}^{(1)}(t) \psi_{j}^{(1)}(s)
$$

be the singular value decomposition (SVD) of $k_{1}(t, s)$. Since the integral operator $K_{1}$ with the kernel $k_{1}(t, s)$ is of Hilbert-Schmidt type, such a decomposition exists and has the following optimal property. Let $K_{1}^{(\alpha)}$ denote the integral operator with the kernel

$$
k_{1}^{(\alpha)}(t, s)=\sum_{j=1}^{\alpha} \sigma_{j}^{(1)} \phi_{j}^{(1)}(t) \psi_{j}^{(1)}(s)
$$

Then $\left\|K_{1}-K_{1}^{(\alpha)}\right\|=\sigma_{\alpha+1}$ minimizes the distance between $K_{1}$ and any finite rank operator whose rank does not exceed $\alpha$. Here \|\| denotes the operator norm on $L_{[a, b]}^{2}$, see [12], Chapter 6 . In this sense $k_{1}^{(\alpha)}(t, s)$ provides the best approximation to $k_{1}(t, s)$. Moreover, if $k_{1}(t, s) \in C_{[a, b] \times[a, b]}^{p}$, then $\sigma_{n}=O\left(n^{-p}\right)$. In a similar way $k_{2}(t, s)$ is approximated with

$$
k_{2}^{(\alpha)}(t, s)=\sum_{j=1}^{\alpha} \sigma_{j}^{(2)} \phi_{j}^{(2)}(t) \psi_{j}^{(2)}(s)
$$

Consider now the approximate integral equation

$$
x(t)+\sum_{j=1}^{\alpha} \sigma_{j}^{(1)} \phi_{j}^{(1)}(t) \int_{a}^{t} \psi_{j}^{(1)}(s) x(s) d s+\sum_{j=1}^{\alpha} \sigma_{j}^{(2)} \phi_{j}^{(2)}(t) \int_{t}^{b} \psi_{j}^{(2)}(s) x(s) d s=y(t) .
$$

Applying the Clenshaw-Curtis quadrature to this equation, we get

$$
x+\left[\sum_{j=1}^{\alpha} \sigma_{j}^{(1)} D_{\phi_{j}^{(1)}} W D_{\psi_{j}^{(1)}}+\sum_{j=1}^{\alpha} \sigma_{j}^{(2)} D_{\phi_{j}^{(2)}} V D_{\psi_{j}^{(2)}}\right] x=y .
$$

Here $x$ denotes the array of values of $x(t)$ at Chebyshev mesh points, and $D_{a}, a=$ $\left[a_{1}, \ldots, a_{n}\right]$, denotes the diagonal matrix whose diagonal elements are $a_{1}, \ldots, a_{n}$. A direct calculation shows that

$$
\sum_{j=1}^{\alpha} \sigma_{j}^{(1)} D_{\phi_{j}^{(1)}} W D_{\psi_{j}^{(1)}}=\left(\sum_{j=1}^{\alpha} \sigma_{j} \phi_{j}^{(1)} \psi_{j}^{(1)^{T}}\right) \circ W,
$$

and similarly

$$
\sum_{j=1}^{\alpha} \sigma_{j}^{(2)} D_{\phi_{j}^{(2)}} W D_{\psi_{j}^{(2)}}=\left(\sum_{j=1}^{\alpha} \sigma_{j} \phi_{j}^{(2)} \psi_{j}^{(2)^{T}}\right) \circ V
$$


where $\circ$ denotes the Schur matrix product defined in the previous section. With the notation

$$
K_{i}^{\alpha}=\sum_{j=1}^{\alpha} \sigma_{j}^{(i)} \phi_{j}^{(i)} \psi_{j}^{(i)^{T}}, \quad i=1,2,
$$

we can rewrite (14) as follows:

$$
x+\left[K_{1}^{\alpha} \circ W+K_{2}^{\alpha} \circ V\right] x=y,
$$

which has the same form as the equation (12) of the previous section. However, (12) has a clear advantage over (15), as it uses values of $k_{i}(t, s)$ rather than the values of the corresponding semi-separable approximations $k_{i}^{(\alpha)}(t, s)$, and more importantly, it avoids the costly numerical SVD. In fact, the realization that the SVD approximation combined with the Clenshaw-Curtis quadrature gives (15) leads us to the much simpler and more accurate discretization of (12).

\section{The Composite RUle}

In this section, we describe the composite rule corresponding to the quadrature of (12). Let

$$
a=b_{0} \leq b_{1} \leq \cdots \leq b_{m}=b
$$

be a partition of the interval $[a, b]$, and let

$$
\tau_{k}^{(j)}=\frac{1}{2}\left(b_{j}-b_{j-1}\right) \tau_{k}+\frac{1}{2}\left(b_{j}+b_{j-1}\right), \quad k=0,1, \ldots, n_{j},
$$

be the Chebyshev support points mapped into $\left[b_{j-1}, b_{j}\right]$. Define

$$
x(t)=\left\{\begin{array}{cll}
x_{1}(t) & \text { if } b_{0} \leq t \leq b_{1}, \\
x_{2}(t) & \text { if } b_{1}<t \leq b_{2}, \\
\vdots & & \\
x_{m}(t) & \text { if } & b_{m-1}<t \leq b_{m},
\end{array}\right.
$$

and

$$
y(t)=\left\{\begin{array}{cll}
y_{1}(t) & \text { if } \quad b_{0} \leq t \leq b_{1}, \\
y_{2}(t) & \text { if } \quad b_{1}<t \leq b_{2}, \\
\vdots & & \\
y_{m}(t) & \text { if } & b_{m-1}<t \leq b_{m},
\end{array}\right.
$$

and rewrite the equation (1) as a system of $m$ equations, for $j=1, \ldots, m$,

$$
\begin{gathered}
x_{j}(t)+\int_{b_{0}}^{b_{1}} k_{1}(t, s) x_{1}(s) d s+\cdots+\int_{b_{j-1}}^{t} k_{1}(t, s) x_{j}(s) d s+\int_{t}^{b_{j}} k_{2}(t, s) x_{j}(s) d s \\
+(16) \quad+\cdots+\int_{b_{m-1}}^{b_{m}} k_{2}(t, s) x_{m}(s) d s=y_{j}(t) .
\end{gathered}
$$

Applying the quadrature of (12) to each of the integrals, we obtain a system of linear equations as follows, for $j=1, \ldots, m$ :

$$
\begin{array}{r}
\frac{b_{1}-b_{0}}{2}\left[(\mathbf{W}+\mathbf{V}) \circ \mathbf{K}_{1 j}\right] \bar{x}_{1}+\cdots+\left[\mathbf{I}+\frac{b_{j}-b_{j-1}}{2}\left(\mathbf{W} \circ \mathbf{K}_{j j}+\mathbf{V} \circ \tilde{\mathbf{K}}_{j j}\right)\right] \bar{x}_{j} \\
+\cdots+\frac{b_{m}-b_{m-1}}{2}\left[(\mathbf{W}+\mathbf{V}) \circ \mathbf{K}_{m j}\right] \bar{x}_{m}=\bar{y}_{j},
\end{array}
$$


where

$$
\begin{aligned}
\bar{x}_{j} & =\left[x\left(\tau_{0}^{(j)}\right), x\left(\tau_{1}^{(j)}\right), \ldots, x\left(\tau_{n_{j}}^{(j)}\right)\right]^{T}, \quad \bar{y}_{j}=\left[y\left(\tau_{0}^{(j)}\right), y\left(\tau_{1}^{(j)}\right), \ldots, y\left(\tau_{n_{j}}^{(j)}\right)\right]^{T}, \\
\mathbf{K}_{j j} & =\left(k_{1}\left(\tau_{p}^{(j)}, \tau_{q}^{(j)}\right)\right)_{p, q=0}^{n_{j}}, \quad \tilde{\mathbf{K}}_{j j}=\left(k_{2}\left(\tau_{p}^{(j)}, \tau_{q}^{(j)}\right)\right)_{p, q=0}^{n_{j}}, \\
\mathbf{K}_{i j} & =\left(k_{1}\left(\tau_{p}^{(j)}, \tau_{q}^{(i)}\right)\right)_{p, q=0}^{n_{j}, n_{i}}, \quad \text { if } \quad i<j, \\
\mathbf{K}_{i j} & =\left(k_{2}\left(\tau_{p}^{(j)}, \tau_{q}^{(i)}\right)\right)_{p, q=0}^{n_{j}, n_{i}}, \quad \text { if } \quad i>j,
\end{aligned}
$$

or, in a block matrix form,

$$
\left[\begin{array}{cccc}
\mathbf{A}_{11} & \mathbf{A}_{12} & \cdots & \mathbf{A}_{1 m} \\
\mathbf{A}_{21} & \mathbf{A}_{22} & \cdots & \mathbf{A}_{2 m} \\
\vdots & & & \\
\mathbf{A}_{m 1} & \mathbf{A}_{m 2} & \cdots & \mathbf{A}_{m m}
\end{array}\right]\left[\begin{array}{c}
\bar{x}_{1} \\
\bar{x}_{2} \\
\vdots \\
\bar{x}_{m}
\end{array}\right]=\left[\begin{array}{c}
\bar{y}_{1} \\
\bar{y}_{2} \\
\vdots \\
\bar{y}_{m}
\end{array}\right]
$$

where

$$
\begin{aligned}
& \mathbf{A}_{j j}=\left[\mathbf{I}+\frac{b_{j}-b_{j-1}}{2}\left(\mathbf{W} \circ \mathbf{K}_{j j}+\mathbf{V} \circ \tilde{\mathbf{K}}_{j j}\right)\right], \\
& \mathbf{A}_{i j}=\frac{b_{j}-b_{j-1}}{2}\left[(\mathbf{W}+\mathbf{V}) \circ \mathbf{K}_{j i}\right], \quad \text { if } \quad i \neq j .
\end{aligned}
$$

Remark 1. In this paper we do not consider the issue of how to partition the interval $[a, b]$. An adaptive quadrature rule is possible here along the same lines as in [13], [23], namely, by using the size of the last Chebyshev coefficients of $k_{1}, k_{2}$ and $y$ in a given subinterval of the partition to determine whether this subinterval should be further subdivided. This adaptive rule is a part of our research project in which we are going to compare the algorithm of the present paper with existing algorithms for Schrödinger equations with nonlocal potentials.

Remark 2. In general, the matrix (17) is not structured, and is solved by standard Gaussian elimination at the cost of $O\left(\mathrm{~m}^{3}\right)$ arithmetic operations (we assume here that $m$ is much larger than the $n_{j}$ 's). If, however, the semi-smooth kernel $k(t, s)$ has some additional structure, then this structure is usually inherited by the matrix in (17). For example, if $k_{1}$ and $k_{2}$ are low rank kernels, then the matrix $A$ becomes semi-separable, and can be solved by existing linear complexity algorithms. We remark that in the case of the Schrödinger equation with nonlocal potentials discussed in Section 6 below, the overall kernel is obtained as the composition of the semi-separable Green function with nonlocal potential. If the nonlocal potential is also semi-separable, which is the case when the nonlocality arises from exchange terms, then the overall kernel is semi-separable as well, and the numerical techniques presented here, although still applicable, can be replaced by the methods of 15] and [13. These methods give highly accurate linear complexity algorithms for the integral equation itself.

If the kernel $k(t, s)$ depends on the difference of the arguments,

$$
k(t, s)=k(|t-s|)= \begin{cases}k_{1}(t-s) & \text { if } 0 \leq s \leq t \\ k_{2}(t-s) & \text { if } t<s \leq T\end{cases}
$$

and if we use a uniform partition with the same number of points per partition, then

$$
k_{r}\left(\tau_{p}^{(i)}, \tau_{q}^{(i)}\right)=k_{r}\left(\tau_{p}-\tau_{q}\right), \quad r=1,2
$$


and we obtain a block Toeplitz matrix,

$$
\left[\begin{array}{ccccc}
\mathbf{A}_{1} & \tilde{\mathbf{A}}_{2} & \tilde{\mathbf{A}}_{3} & \cdots & \tilde{\mathbf{A}}_{m} \\
\mathbf{A}_{2} & \mathbf{A}_{1} & \tilde{\mathbf{A}}_{2} & \cdots & \tilde{\mathbf{A}}_{m-1} \\
\mathbf{A}_{3} & \mathbf{A}_{2} & \mathbf{A}_{1} & \cdots & \tilde{\mathbf{A}}_{m-2} \\
& \ddots & \ddots & \ddots & \\
\mathbf{A}_{m} & \mathbf{A}_{m-1} & \cdots & \mathbf{A}_{2} & \mathbf{A}_{1}
\end{array}\right]\left[\begin{array}{c}
\bar{x}_{1} \\
\bar{x}_{2} \\
\vdots \\
\bar{x}_{m}
\end{array}\right]=\left[\begin{array}{c}
\bar{y}_{1} \\
\bar{y}_{2} \\
\vdots \\
\bar{y}_{m}
\end{array}\right] .
$$

This Toeplitz system of equations can be efficiently solved by the iterative conjugate gradients methods in $O(m \log (m))$ arithmetic operations, or by direct divideand-conquer type algorithms in $O\left(m \log ^{2}(m)\right)$ arithmetic operations, or by direct Levinson type algorithms in $O\left(\mathrm{~m}^{2}\right)$ arithmetic operations. Each of these techniques has its uses, depending on the properties of the Toeplitz matrix and the underlying science model. References to Toeplitz solvers can be found, for example, in an expository paper by Chan and $\mathrm{Ng}$ [6]. We did some preliminary numerical experiments with the Wiener-Hopf integral equation

$$
x(t)+\int_{0}^{\infty} \frac{1}{1+|t-s|^{3}} x(s) d s=e^{-t}, \quad 0 \leq t \leq \infty .
$$

With the truncation limit at $t=32$, and with 128 partitions with 4 points per partition, the NCC quadrature combined with the block-Levinson algorithm for block Toeplitz matrices gave the accuracy of $2.43 e-07$. The best accuracy reached by Gaussian elimination for 64 partitions with 4 points, per partition was $8.47 e-$ 0.5. For 128 partitions the application of Gaussian elimination became impossible because the CPU time limit was exceeded. Our study of the application of the NCC quadrature to Wiener-Hopf type equations in combination with appropriate direct and iterative Toeplitz solvers is now in progress.

Remark 3. Numerical examples in the next section demonstrate that the NCC quadrature shows good accuracy when the semi-smooth kernel has some singularities on the boundary of the square $[a, b] \times[a, b]$, e.g., see Example 3. The kernels with a "running" singularity along the diagonal $t=s$, like $k(t, s)=|t-s|^{-1}$, are not semi-smooth, and hence the NCC quadrature is not applicable to such kernels. If, however, the singularities on the diagonal occur at a finite number of isolated points, then the adaptive NCC quadrature which has all the singular points as end-points of partitions is applicable, since the restriction of the kernel onto each of the subrectangles of the partition is semi-smooth with possible singularities on the boundary only. Example 4 in the next section illustrates this point.

\section{NumERICAL EXAMPLES}

In this section we compare our methods with some existing algorithms for the following types of kernels:

- Type 1: Discontinuity along the diagonal $t=s$.

- Type 2: Discontinuity in the first order partial derivatives along the diagonal $t=s$.

- Type 3: Singularity on the boundary of the square and Type 2.

- Type 4: Singularity on the main diagonal.

These are the methods which have been implemented for comparison purposes:

G-Leg: Nyström quadrature based on the Gauss-Legendre rule. 
T-Def: Two step deferred approach to the limit. Approximate solutions $x_{1}$, $x_{2}$, and $x_{3}$ for subintervals of partition $h, \frac{h}{2}$, and $\frac{h}{4}$, respectively, are computed. Then the numerical solution $x(s)$ is obtained by (see, e.g., Baker [5])

$$
x(s)=\frac{64 x_{3}(s)+x_{1}(s)-20 x_{2}(s)}{45} .
$$

Atk-T: Atkinson's iteration with the composite trapezium rule, applied to kernels $k(t, s)$ which have discontinuities in the first order partial derivatives along the diagonal $t=s$.

Alg-1: Algorithm of Section 2, (3).

NCC: Algorithm of Section 3, (12).

NCC-C: Algorithm of Section 4, (17).

The number of points used in discretizations is denoted by $n$. Error denotes $\left\|x-x_{\tau}\right\| /\|x\|$, where $x$ and $x_{\tau}$ are the analytic and the numerical solutions, respectively. In each plot, $\log$ (Error) is the common logarithm of the Error. All computations were done on a DELL Workstation with operating system RedHat Linux 5.2 in double precision. All examples are set up by choosing a simple analytic solution and then computing the corresponding right hand side. We remark that the values of $x(t)$ are found inside the interval (or each of the subintervals of partition) at Chebyshev points $\tau_{0}, \tau_{1}, \ldots, \tau_{n}$. The value of $x(t)$ for $t \neq \tau_{k}$ can be found as follows. Applying $\mathbf{C}^{-1}$, we can find "Chebyshev-Fourier" coeffcients of $x(t)$,

$$
\left[\begin{array}{c}
\alpha_{0} \\
\alpha_{1} \\
\vdots \\
\alpha_{n}
\end{array}\right]=\mathbf{C}^{-1}\left[\begin{array}{c}
x\left(\tau_{0}\right) \\
x\left(\tau_{1}\right) \\
\vdots \\
x\left(\tau_{n}\right)
\end{array}\right]
$$

Thus,

$$
x(t) \cong \sum_{j=0}^{n} \alpha_{j} T_{j}(h(t)), \quad a \leq t \leq b .
$$

The value of $T_{j}(t)$ for $t \neq \tau_{k}$ is now found using the recursion satisfied by Chebyshev polynomials, $T_{j+1}(t)=2 t T_{j}(t)-T_{j-1}(t)$.

\section{Example 1.}

$$
x(t)+\lambda \int_{-1}^{1} k(t, s) x(s) d s=y(t), \quad-1 \leq t \leq 1,
$$

where $y(t)=\lambda\left(e+e^{-1}\right)+(1-2 \lambda) e^{-t}$, and

$$
k(t, s)=\left\{\begin{array}{c}
1 \quad \text { if } \quad-1 \leq s \leq t \\
-1 \quad \text { if } \quad t<s \leq 1
\end{array}\right.
$$

The analytical solution is $x(t)=e^{-t}$. Since this kernel is discontinuous along the diagonal $t=s$, Gauss-Legendre quadrature gives low accuracy. The accuracy in the Atkinson's iteration improves very slowly. The algorithm of Section 3 gives accuracy of order $10^{-15}$ with only 16 support points, whereas the 2 -step method of deferred approach to the limit requires $n=256$ points to achieve comparable accuracy (see Figure 1). Moreover, it requires computation of $x_{2}(t)$ and $x_{3}(t)$ at the cost of $O\left((2 n)^{3}\right)$ and $O\left((4 n)^{3}\right)$, respectively. 


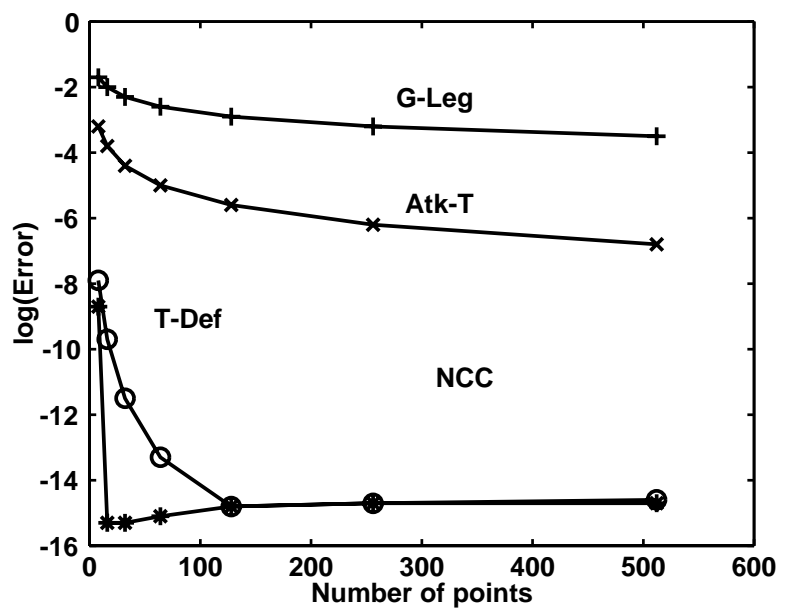

Figure 1. Comparison of numerical solutions of Example 1, $\lambda=0.1$.

\section{Example 2.}

$$
x(t)+\lambda \int_{0}^{T} k(t, s) x(s) d s=y(t), \quad 0 \leq t \leq T,
$$

where $y(t)=\left(1-\frac{\lambda \sin ^{2}(T)}{2}+\lambda\right) \sin (t)+\left(\frac{T}{2}-t-\frac{\sin (2 T)}{4}\right) \lambda \cos (t)$, and

$$
k(t, s)=\sin (|t-s|)= \begin{cases}\sin (t-s) & \text { if } 0 \leq s \leq t \\ \sin (s-t) & \text { if } t<s \leq \frac{\pi}{2}\end{cases}
$$

The analytical solution is $x(t)=\sin (t)$. This kernel has discontinuities in the first order partial derivatives along the diagonal, $t=s$. Again standard Nyström-type discretization methods fail to give high accuracy in this case. In the first experiment we take $T=\frac{\pi}{2}$ and $\lambda=-\frac{4}{\pi}$. Our method shows accuracy of order $10^{-14}$ with only 16 points in $\left[0, \frac{\pi}{2}\right]$ without any partitioning. The 2 -step method of the deferred approach to the limit gives accuracy $O\left(10^{-14}\right)$ with $n=256$, but at much higher cost than our method (see Figure 2). The second part of Example 2 is to compare the composite rule described in Section 4 with the basic quadrature (12) of Section 3 when the length of the interval of integration $[a, b]$ becomes increasingly large. Here $M$ denotes the number of subintervals in $[0, T]$, and $M n$ stands for the total number of support points in $[0, T]$.

Without partitioning, i.e., with $M=1$, we increase the number of support points from $n=128$ to $n=1024$. For $n=512$ the accuracy is of order $O\left(10^{-2}\right)$, but for $n=1024$ the CPU time limit is exceeded. When the interval is partitioned into

$$
\text { TABLE 1. }\left(T=200 \pi, \quad \lambda=-\frac{4}{\pi}\right)
$$

\begin{tabular}{|c|c|c|c|c|c|c|}
\hline$M$ & 1 & 1 & 1 & 2 & 4 & 8 \\
\hline$M n$ & 256 & 512 & 1024 & 256 & 512 & 1024 \\
\hline Error & $2.4 e+01$ & $3.0 e-02$ & CPU time limit exceed & $1.2 e+01$ & $7.8 e-02$ & $2.2 e-11$ \\
\hline
\end{tabular}






FiguRE 2. Comparison of numerical solutions of Example 2.

8 subintervals and $n=128$, i.e., the total number of points is 1024 , the accuracy now is of order $O\left(10^{-11}\right)$ (see Table 1).

\section{Example 3.}

$$
x(t)+\int_{-1}^{1} k(t, s) x(s) d s=y(t), \quad-1 \leq t \leq 1,
$$

where $y(t)=1-t^{2}+\frac{1}{1-t^{2}}(\arctan (t)-\arctan (-1))-\frac{1}{(1+t)\left(1+t^{2}\right)}$, and

$$
k(t, s)= \begin{cases}\frac{1}{\left(1-t^{2}\right)\left(1-s^{4}\right)} & \text { if }-1 \leq s \leq t \\ \frac{-1}{\left(1-t^{4}\right)\left(1-s^{2}\right)} & \text { if } t<s \leq 1\end{cases}
$$

The analytical solution is $x(t)=1-t^{2}$. Since this kernel has singularities along the boundaries of the square $[-1,1] \times[-1,1]$, methods based on the trapezium rule are not applicable. Therefore we compare our algorithms of Section 2 and Section 3 with the Nyström-Gauss-Legendre discretization only. The algorithm of Section 2 shows the same accuracy of numerical solution as the Gauss-Legendre quadrature. The method of Section 3 gives $O\left(10^{-13}\right)$ accuracy with $n=32$ points, whereas Nyström-Gauss-Legendre quadrature gives $O\left(10^{-3}\right)$ with $n=256$ points (see Figure 3).

\section{Example 4.}

$$
x(t)+\int_{-1}^{1} k(t, s) x(s) d s=y(t), \quad-1 \leq t \leq 1,
$$

where $y(t)=2\left(1-t^{2}+2 t^{3}\right)+\left(1+2 t^{4}\right) \ln \left(t^{2}+t^{4}\right)-\ln \left(1+t^{2}\right)-2 t^{4} \ln \left(1+t^{4}\right)$, and

$$
k(t, s)= \begin{cases}1 /\left(t^{2}+s^{4}\right) & \text { if }-1 \leq s \leq t \\ 1 /\left(s^{2}+t^{4}\right) & \text { if } t<s \leq 1\end{cases}
$$

The analytical solution is $x(t)=4 t^{3}$. The kernel $k(t, s)$ has a singularity at $(0,0)$. Also $y(t)$ is singular at $t=0$. Therefore we partition $[-1,1]$ into $[-1,0]$ and $[0,1]$. The choice of $n=256$ Chebyshev points in each subinterval with the total of $n=512$ points gives $O\left(10^{-11}\right)$ accuracy. For comparison, the best accuracy of the 


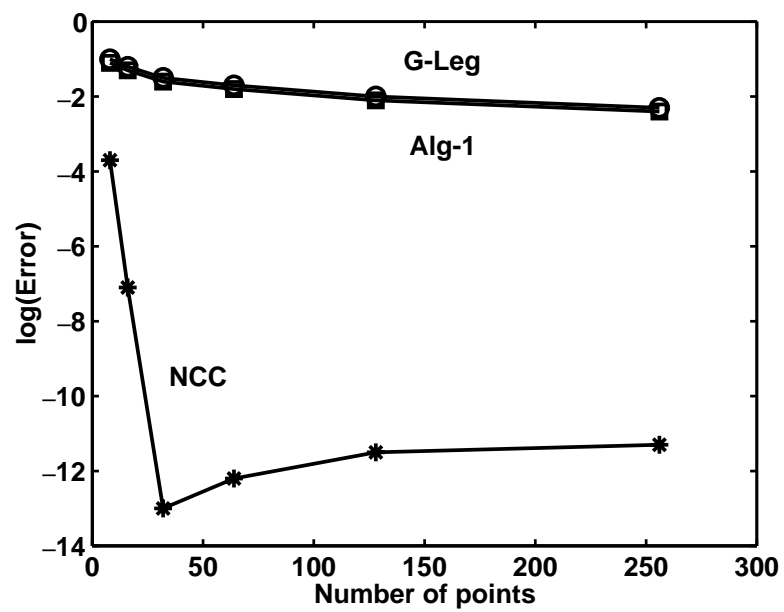

Figure 3. Comparison of numerical solutions of Example 3.

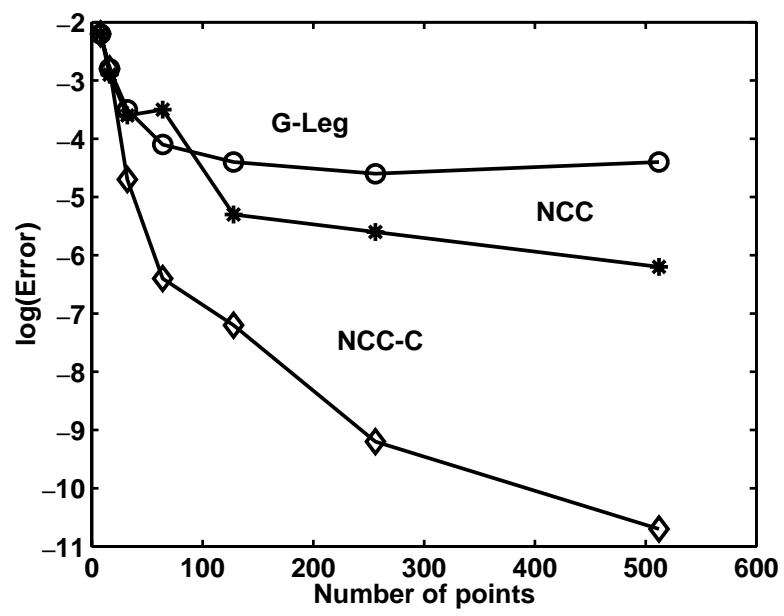

Figure 4. Comparison of numerical solutions of Example 4.

Gauss-Legendre quadrature without partitions and with $n=512$ support points is $O\left(10^{-4}\right)$, while the best accuracy of the algorithm of Section 3 without partitions is $O\left(10^{-6}\right)$ (see Figure 4).

\section{Application to nONLOCAl SchröDinger EQUations}

In this section we demonstrate that the developed numerical technique is also applicable to problems other than integral equations, for example, to integrodifferential equations. We chose here the radial Schrödinger equation which models the quantum mechanical interaction between particles represented by spherically symmetric potentials. These potentials are usually local, i.e., they depend only on the distance between the two particles, in which case the equation is a differential equation which is routinely solved in computational physics. However, if there are 
more than two particles present, then the potentials can become nonlocal and the differential Schrödinger equation becomes an integro-differential equation for the wave function $\psi$,

$$
\frac{d^{2} \psi(r)}{d r^{2}}+\kappa^{2} \psi(r)=\int_{0}^{T} v\left(r, r^{\prime}\right) \psi\left(r^{\prime}\right) d r^{\prime}
$$

which is defined for $0<r<\infty$, satisfies the condition $\psi(0)=0$, and is bounded at infinity. It is assumed that $v\left(r, r^{\prime}\right)$ is negligible for $r>T$ or $r^{\prime}>T$, see, e.g., 21]. Because it is numerically more difficult to solve the Schrödinger equation in the presence of a nonlocal potential, the latter is customarily replaced by an approximate local equivalent potential. There is, however, a renewed interest in the nonlocal equations, and a significant number of papers on this subject appeared in the past few years (our database search returned over 50 related publications).

Using the technique of [13], it easy to show that (20) is equivalent to the following integral equation:

$$
\begin{aligned}
\psi(r) & +\frac{\cos (\kappa r)}{\kappa} \int_{0}^{r} \sin \left(\kappa r^{\prime}\right) \int_{0}^{T} v\left(r^{\prime}, p\right) \psi(p) d p d r^{\prime} \\
& +\frac{\sin (\kappa r)}{\kappa} \int_{r}^{T} \cos \left(\kappa r^{\prime}\right) \int_{0}^{T} v\left(r^{\prime}, p\right) \psi(p) d p d r^{\prime}=\sin (\kappa r) .
\end{aligned}
$$

or

$$
\begin{gathered}
\psi(r)+\frac{\cos (\kappa r)}{\kappa} \int_{0}^{T} k_{1}\left(r, r^{\prime}\right) \psi\left(r^{\prime}\right) d r^{\prime}+\frac{\sin (\kappa r)}{\kappa} \int_{0}^{T} k_{2}\left(r, r^{\prime}\right) \psi\left(r^{\prime}\right) d r^{\prime} \\
=\sin (\kappa r),
\end{gathered}
$$

where

$$
k_{1}\left(r, r^{\prime}\right)=\int_{0}^{r} \sin (\kappa p) v\left(p, r^{\prime}\right) d p, \quad k_{2}\left(r, r^{\prime}\right)=\int_{r}^{T} \cos (\kappa p) v\left(p, r^{\prime}\right) d p .
$$

We consider now the case when $v\left(p, r^{\prime}\right)$ is semi-smooth, such that

$$
v\left(p, r^{\prime}\right)= \begin{cases}v_{1}\left(p, r^{\prime}\right) & \text { if } 0 \leq p \leq r^{\prime} \\ v_{2}\left(p, r^{\prime}\right) & \text { if } r^{\prime} \leq p \leq T .\end{cases}
$$

In order to use the method which we developed in previous sections, we rewrite equation (21) as follows:

$$
\begin{aligned}
& \psi(r)+\frac{c(r)}{\kappa} \int_{0}^{r} k_{1}\left(r, r^{\prime}\right) \psi\left(r^{\prime}\right) d r^{\prime}+\frac{c(r)}{\kappa} \int_{r}^{T} k_{1}\left(r, r^{\prime}\right) \psi\left(r^{\prime}\right) d r^{\prime} \\
& +\frac{s(r)}{\kappa} \int_{0}^{r} k_{2}\left(r, r^{\prime}\right) \psi\left(r^{\prime}\right) d r^{\prime}+\frac{s(r)}{\kappa} \int_{r}^{T} k_{2}\left(r, r^{\prime}\right) \psi\left(r^{\prime}\right) d r^{\prime}=s(r),
\end{aligned}
$$

where for notational convenience we abbreviate $c(r)=\cos (\kappa r), s(r)=\sin (\kappa r)$. We have

$$
k_{1}\left(r, r^{\prime}\right)= \begin{cases}k_{11}\left(r, r^{\prime}\right) & \text { if } 0 \leq r^{\prime} \leq r \\ k_{12}\left(r, r^{\prime}\right) & \text { if } 0 \leq r \leq r^{\prime}\end{cases}
$$

and

$$
k_{2}\left(r, r^{\prime}\right)= \begin{cases}k_{21}\left(r, r^{\prime}\right) & \text { if } r^{\prime} \leq r \leq T \\ k_{22}\left(r, r^{\prime}\right) & \text { if } r \leq r^{\prime} \leq T\end{cases}
$$


where

$$
\begin{aligned}
k_{11}\left(r, r^{\prime}\right) & =\int_{0}^{r^{\prime}} s(p) v_{1}\left(p, r^{\prime}\right) d p+\int_{r^{\prime}}^{r} s(p) v_{2}\left(p, r^{\prime}\right) d p \\
& =\int_{0}^{r^{\prime}} s(p) v_{1}\left(p, r^{\prime}\right) d p+\int_{0}^{r} s(p) v_{2}\left(p, r^{\prime}\right) d p-\int_{0}^{r^{\prime}} s(p) v_{2}\left(p, r^{\prime}\right) d p \\
k_{12}\left(r, r^{\prime}\right) & =\int_{0}^{r} s(p) v_{1}\left(p, r^{\prime}\right) d p \\
k_{21}\left(r, r^{\prime}\right) & =\int_{r}^{T} c(p) v_{2}\left(p, r^{\prime}\right) d p \\
k_{22}\left(r, r^{\prime}\right) & =\int_{r}^{r^{\prime}} c(p) v_{1}\left(p, r^{\prime}\right) d p+\int_{r^{\prime}}^{T} c(p) v_{2}\left(p, r^{\prime}\right) d p \\
& =\int_{r}^{T} c(p) v_{1}\left(p, r^{\prime}\right) d p-\int_{r^{\prime}}^{T} c(p) v_{1}\left(p, r^{\prime}\right) d p+\int_{r^{\prime}}^{T} c(p) v_{2}\left(p, r^{\prime}\right) d p
\end{aligned}
$$

Thus,

$$
\begin{aligned}
& \psi(r)+\frac{c(r)}{\kappa} \int_{0}^{r} k_{11}\left(r, r^{\prime}\right) \psi\left(r^{\prime}\right) d r^{\prime}+\frac{c(r)}{\kappa} \int_{r}^{T} k_{12}\left(r, r^{\prime}\right) \psi\left(r^{\prime}\right) d r^{\prime} \\
& +\frac{s(r)}{\kappa} \int_{0}^{r} k_{21}\left(r, r^{\prime}\right) \psi\left(r^{\prime}\right) d r^{\prime}+\frac{s(r)}{\kappa} \int_{r}^{T} k_{22}\left(r, r^{\prime}\right) \psi\left(r^{\prime}\right) d r^{\prime}=s(r) .
\end{aligned}
$$

Applying our quadrature to this equation, we get

$$
\left[\mathbf{I}+\frac{T}{2 \kappa} \mathbf{D}_{c}\left(\mathbf{W} \circ \mathbf{K}_{11}+\mathbf{V} \circ \mathbf{K}_{12}\right)+\frac{T}{2 \kappa} \mathbf{D}_{s}\left(\mathbf{W} \circ \mathbf{K}_{21}+\mathbf{V} \circ \mathbf{K}_{22}\right)\right] \bar{\psi}=\overline{\mathbf{s}},
$$

where, in more detail,

$$
\begin{aligned}
\bar{\psi} & =\left[\psi\left(t_{0}\right), \psi\left(t_{1}\right), \ldots, \psi\left(t_{n}\right)\right]^{T}, \\
\mathbf{D}_{c} & =\operatorname{diag}\left(\cos \left(\kappa t_{0}\right), \cos \left(\kappa t_{1}\right), \ldots, \cos \left(\kappa t_{n}\right)\right), \\
\mathbf{D}_{s} & =\operatorname{diag}\left(\sin \left(\kappa t_{0}\right), \sin \left(\kappa t_{1}\right), \ldots, \sin \left(\kappa t_{n}\right)\right), \\
\mathbf{D}_{\sigma} & =\operatorname{diag}\left([1, \ldots, 1] \mathbf{S}_{L} \mathbf{C}^{-1}\right), \\
\overline{\mathbf{s}} & =\left[\sin \left(\kappa t_{0}\right), \sin \left(\kappa t_{1}\right), \ldots, \sin \left(\kappa t_{n}\right)\right]^{T}, \\
\mathbf{W} & =\mathbf{C S}_{L} \mathbf{C}^{-1}, \mathbf{V}=\mathbf{C S}_{R} \mathbf{C}^{-1}, \\
\mathbf{K}_{11} & =\left(k_{11}\left(t_{i}, t_{j}\right)\right)_{i, j=0}^{n} \\
& =\frac{T}{2}\left[\operatorname{diag}\left(\mathbf{W D}_{s}\left(\mathbf{V}_{1}-\mathbf{V}_{2}\right)\right)+\left(\mathbf{W D}_{s} \mathbf{V}_{2}\right)\right], \\
\mathbf{K}_{12} & =\left(k_{12}\left(t_{i}, t_{j}\right)\right)_{i, j=0}^{n} \\
& =\frac{T}{2}\left(\mathbf{W D}_{s} \mathbf{V}_{1}\right), \\
\mathbf{K}_{21} & =\left(k_{21}\left(t_{i}, t_{j}\right)\right)_{i, j=0}^{n}, \\
& =\frac{T}{2}\left(\mathbf{V D}_{c} \mathbf{V}_{2}\right) \\
\mathbf{K}_{22} & =\left(k_{22}\left(t_{i}, t_{j}\right)\right)_{i, j=0}^{n} \\
& =\frac{T}{2}\left[\left(\mathbf{V D}_{c} \mathbf{V}_{1}\right)+\operatorname{diag}\left(\mathbf{V D}_{c}\left(\mathbf{V}_{2}-\mathbf{V}_{1}\right)\right)\right] .
\end{aligned}
$$


Now we illustrate our discretization with examples. In the first example we use a prototype of the Yukawa potential, (e.g., [18], 23.c), which is simplified to a degree such that an analytic solution can be found. In our terminology this potential is semi-separable. We note once more that the case of this semi-separable potential could be treated more easily by the techniques already presented in [13], and we use it here only because the comparison with the analytic solution is possible.

Example 1. Let

$$
v\left(p, r^{\prime}\right)= \begin{cases}\lambda e^{p-r^{\prime}} & \text { if } 0 \leq p \leq r^{\prime} \\ \lambda e^{r^{\prime}-p} & \text { if } r^{\prime} \leq p \leq T\end{cases}
$$

It is easy to see that if $\psi(r)=e^{-r}$, then the right-hand side has the form

$$
y(r)=\left(1-\frac{3 \lambda \kappa}{4}\right) e^{-r}+\frac{3 \lambda \kappa}{4} \cos (r)-\frac{\lambda \kappa}{2} r e^{-r} .
$$

By comparing the analytical solution given above with the numerical solution of (24) at the discretization points, we get the following relative errors in the case of $\lambda=0.1, \quad \kappa=1$ and $T=20$.

\begin{tabular}{|c|c|c|c|c|c|}
\hline$n$ & 16 & 32 & 64 & 128 & 256 \\
\hline Error & $1.2 e+01$ & $3.4 e-07$ & $8.1 e-09$ & $3.4 e-09$ & $6.0 e-09$ \\
\hline
\end{tabular}

In the second example we consider a more interesting case for which the techniques of [13] are not applicable. This time the nonlocality is a prototype of the optical model Perey-Buck potential, see the Appendix for details. In our terminology this potential is semi-smooth, but not semi-separable.

Example 2. Let

$$
v\left(p, r^{\prime}\right)=\frac{\lambda e^{-\frac{\left|r^{\prime}-p\right|}{A}}}{1+e^{-\frac{\left|r^{\prime}-p\right|}{A}}}=\left\{\begin{array}{lll}
\frac{\lambda e^{\frac{p-r^{\prime}}{A}}}{1+e^{\frac{p-r^{\prime}}{A}}} & \text { if } & 0 \leq p \leq r^{\prime}, \\
\frac{\lambda e^{\frac{r^{\prime}-p}{A}}}{1+e^{\frac{r^{\prime}-p}{A}}} & \text { if } & r^{\prime} \leq p \leq T .
\end{array}\right.
$$

Solving (24) at $n$ shifted Chebyshev support points $t_{i}^{(n)}, i=1, \ldots, n$, and $2 n$ points $s_{i}^{(2 n)}, i=1, \ldots, 2 n$, we obtain numerical solutions $\psi^{(n)}(r)$ and $\psi^{(2 n)}(r)$, respectively.

To get the values of $\psi^{(2 n)}(r)$ at $t_{i}^{(n)}$, we follow the procedure described in the beginning of Section 5. The error $e_{n}$ is obtained by comparison of the solutions $\psi^{(n)}$ and $\psi^{(2 n)}$ as follows:

$$
e_{n}=\left\|\psi^{(2 n)}\left(t_{i}^{(n)}\right)-\psi^{(n)}\left(t_{i}^{(n)}\right)\right\|_{\infty} /\left\|\psi^{(2 n)}\left(t_{i}^{(n)}\right)\right\|_{\infty} .
$$

Here we take $\lambda=0.1, \kappa=1, \quad A=100$, and $T=20$.

\begin{tabular}{|c|c|c|c|c|c|c|}
\hline$n$ & 8 & 16 & 32 & 64 & 128 & 256 \\
\hline$e_{n}$ & $1.0 e-0$ & $1.2 e-03$ & $1.6 e-09$ & $7.7 e-15$ & $1.6 e-14$ & $4.8 e-14$ \\
\hline
\end{tabular}

We see that for this choice of $\lambda$ the discrete equations are well conditioned and the double precision accuracy is obtained with 64 points. 
In the third and fourth examples we consider a more difficult case, which models the nonlocalities corresponding to a nucleon-nucleon interaction as explained in the Appendix. In this case the kernel is continuous, but its first or second derivatives are singular along the main diagonal. These singularities affect spectral accuracy of the NCC quadrature, which now gives only a single precision accuracy.

Example 3. Let

$$
v\left(p, r^{\prime}\right)=\left(p-r^{\prime}\right)^{2} \ln \left(\left(p-r^{\prime}\right)^{2}\right) e^{-\left|p-r^{\prime}\right|} e^{-\left(p+r^{\prime}\right)} .
$$

The right-hand side is chosen to be $y(r)=\sin (r)$. Note that the right hand side is bounded and does not approach zero at infinity, which is typical for nuclear scattering applications. We use $T=14$ and $k=1$. The best accuracy of our algorithm is achieved for the choice of 32 partitions with 16 points per partition with the error of $6.7 e-06$.

Example 4. Let

$$
v\left(p, r^{\prime}\right)=\left|p-r^{\prime}\right| \ln \left(\left(p-r^{\prime}\right)^{2}\right) e^{-\left|p-r^{\prime}\right|} e^{-\left(p+r^{\prime}\right)} .
$$

The right-hand side is chosen to be $y(r)=\sin (r)$, and we use $T=14$ and $k=1$. Again, the best accuracy of our algorithm is achieved for the choice of 32 partitions with 16 points per partition with the error of $5.6 e-04$.

\section{Summary AND CONCLUSIONS}

In this paper, which is one of a sequence treating integral equations, we describe a new accurate discretization technique for integral equations whose kernels can be discontinuous along the main diagonal. It has the following advantages for a large class of such kernels:

(i) For semi-smooth kernels it gives a much higher accuracy than was ever possible with standard Gauss type quadrature rules.

(ii) It is of comparable accuracy with Gauss type quadratures for smooth kernels.

(iii) It exploits additional structure of the kernel such as a low semi-rank, or a displacement structure, for example, to allow for reduced complexity algorithms for the discretized equations.

(iv) The numerical examples provided in the present study illustrate increased accuracy of our method compared to other more conventional methods.

Our method is applicable to the solution of integral equations, to the computation of eigenvalues and eigenfunctions of integral and differential operators, and to the solution of integro-differential equations.

Our method is expected to find applications in quantum mechanical atomic and nuclear physics problems, where the requirement of indistinguishability of the electrons leads to nonlocalities in the potential contained in the Schrödinger equation due to the presence of exchange terms. These, in turn, lead to integro-differential equations which are usually solved by iterative finite difference methods, or by orthogonal function expansion methods. We plan to compare our new method with some of the existing methods in future investigations.

\section{Appendix: Physical examples of nOnlocalities FOR QUANTUM MECHANICAL CALCULATIONS}

9.1. Introduction. The quantum mechanical wave function of a system of particles obeys the Schrödinger wave equation. For the case of a particle moving in a 
force field $\vec{F}(\vec{r})$, where $\vec{r}$ is the position vector of the particle from the origin, the time independent form of the Schrödinger equation is

$$
\left[-\frac{\hbar^{2}}{2 m} \nabla_{\vec{r}}^{2}+V(\vec{r})\right] \Psi(\vec{r})=E \Psi(\vec{r}),
$$

where $E$ is the energy of the particle, $m$ is the mass, $\hbar$ is Planck's constant divided by $2 \pi, V(\vec{r})$ is the potential, so that $\vec{F}(\vec{r})=-\vec{\nabla} V(\vec{r})$, and

$$
\nabla_{\vec{r}}^{2}=\frac{\partial^{2}}{\partial x^{2}}+\frac{\partial^{2}}{\partial y^{2}}+\frac{\partial^{2}}{\partial z^{2}}
$$

is the Laplacian. In the above equation the potential is local. For a nonlocal potential, the term $V(\vec{r}) \Psi(\vec{r})$ is replaced by

$$
V(\vec{r}) \Psi(\vec{r}) \Longrightarrow \int U\left(\vec{r}, \vec{r}^{\prime}\right) \Psi\left(\vec{r}^{\prime}\right) d^{3} \vec{r}^{\prime},
$$

and the equation becomes an integro-differential equation.

Nonlocal potentials can occur in the case of the interaction of one particle with a system of particles. Examples are as follows:

a) The exchange terms required for identical particles so as to obey the Pauli exclusion principle, 21, Chapter 18, lead to nonlocalities usually of a semi-separable form.

b) When explicit reference to some particles is suppressed, and is replaced in terms of expressions involving integrals over Green functions between the remaining particles [10], then the resulting nonlocal potential becomes nonsemi-separable. An example given below refers to the interaction of two nucleons (protons or neutrons), mediated by the exchange of mesons, whose coordinates are suppressed.

c) Phenomenological nonlocalities have also been introduced in the past to simulate effects a) and b) mentioned above. A well known example in nuclear physics is the Perey-Buck nonlocality, 22, used to simulate the energy dependence of an equivalent local potential which describes the scattering of an incident nucleon from a nucleus.

9.2. Reduction to a one dimensional equation. In many applications the potential in (1) or (2) does not depend on the choice of the direction of the axes of the coordinate system. In this case

$$
\begin{gathered}
V(\vec{r})=V(r), \\
U\left(\vec{r}, \vec{r}^{\prime}\right)=U\left(r, r^{\prime}, x\right),
\end{gathered}
$$

where $r=|\vec{r}|$ is the length of the vector $\vec{r}$, and $x$ is the cosine of the angle between $\vec{r}$ and $\vec{r}^{\prime}$. In this case a partial wave expansion of the wave function (e.g., [18]) in terms of spherical harmonics $Y_{l m}(\hat{r})$, where $\hat{r}$ is the unit vector pointing in the direction $\vec{r}$, is given by

$$
\Psi(\vec{r})=\sum_{l} \frac{1}{r} \Re_{l}(r) \sum_{m} A_{l m} Y_{l m}(\theta, \phi), l=0,1,2, \ldots, \quad m=-l,-l+1, \ldots, l .
$$

Since the spherical harmonics are eigenfunctions of a piece of the Laplacian in polar coordinates, the partial wave expansion permits one to reduce the three dimensional 
equation (1) into a sequence of one dimensional equations for the radial partial waves $\Re_{l}(r)$ :

$$
-\frac{\hbar^{2}}{2 m} \frac{d^{2}}{d r^{2}} \Re_{l}(r)+\left[V_{l}-E\right] \Re_{l}(r)+\int_{0}^{\infty} U_{l}\left(r, r^{\prime}\right) \Re_{l}\left(r^{\prime}\right) d r^{\prime}=0,
$$

and the constants $A_{l m}$ are determined from the boundary conditions. The local part of the overall potential is given by

$$
V_{l}(r)=\frac{\hbar^{2}}{2 m} \frac{l(l+1)}{r^{2}}+V(r)
$$

and the nonlocal part is given by

$$
U_{l}\left(r, r^{\prime}\right)=2 \pi r r^{\prime}(2 l+1) \int_{-1}^{1} U\left(r, r^{\prime}, x\right) P_{l}(x) d x,
$$

where $P_{l}(x)$ are the Legendre polynomials.

The result of (31), which is equivalent to equation (18) in [22], is obtained by inserting equation (29) into (25), multiplying (25) by $Y_{l m}^{*}(\theta, \phi)$, integrating over the solid angle $d \Omega$, using the scalar nature of the potentials (27) and (28), and using an addition theorem for the product of two spherical harmonics.

By introducing $\rho=\left|\vec{r}-\vec{r}^{\prime}\right|$, and since

$$
\rho^{2}=\left(\vec{r}-\vec{r}^{\prime}\right)^{2}=r^{2}+\left(r^{\prime}\right)^{2}-2 r r^{\prime} x,
$$

one has $\rho d \rho=-r r^{\prime} d x$, and hence (31) can be written in the form

$$
U_{l}\left(r, r^{\prime}\right)=(2 l+1) 2 \pi \int_{\left|r-r^{\prime}\right|}^{r+r^{\prime}} U\left(r, r^{\prime}, x\right) P_{l}(x) \rho d \rho .
$$

The results of equations (31) and (32) are equivalent to the expansion of a function $F\left(r, r^{\prime}, x\right)$ into Legendre polynomials in the variable $x$,

$$
F\left(r, r^{\prime}, x\right)=\sum_{l}(l+1 / 2) F_{l}\left(r, r^{\prime}\right) P_{l}(x),
$$

which, as a result of the orthogonality of Legendre polynomials, gives

$$
F_{l}\left(r, r^{\prime}\right)=\int_{-1}^{1} F\left(r, r^{\prime}, x\right) P_{l}(x) d x .
$$

A well known example of the above is the expansion of $\left|\vec{r}-\vec{r}^{\prime}\right|^{-1}$,

$$
\frac{1}{\rho}=\sum_{l} C_{l}^{(1)}\left(r, r^{\prime}\right) P_{l}(x)
$$

which, in view of (33) and (34), provides the well known semi-separable representation of the coefficients,

$$
\begin{gathered}
C_{l}^{(1)}=\frac{r^{l}}{\left(r^{\prime}\right)^{l+1}}, \quad r<r^{\prime}, \\
C_{l}^{(1)}=\frac{\left(r^{\prime}\right)^{l}}{r^{l+1}}, \quad r^{\prime}<r .
\end{gathered}
$$

However, the expansion of $1 / \rho^{2}$ has coefficients which are no longer semi-separable. Rather, that expansion,

$$
\frac{1}{\rho^{2}}=\sum_{l} C_{l}^{(2)}\left(r, r^{\prime}\right) P_{l}(x)
$$


has coefficients with logarithmic singularities of the form

$$
\begin{gathered}
C_{0}^{(2)}=\frac{1}{2 r r^{\prime}} \ln \frac{r+r^{\prime}}{\left|r-r^{\prime}\right|}, \\
C_{1}^{(2)}=\frac{3}{2 r r^{\prime}}\left[\frac{r^{2}+\left(r^{\prime}\right)^{2}}{2 r r^{\prime}} \ln \frac{r+r^{\prime}}{\left|r-r^{\prime}\right|}-1\right],
\end{gathered}
$$

etc. Other cases will be treated further on.

The next step is to replace the integro-differential equation (30) by an integral equation, by first writing (30) in the form

$$
\left(\frac{d^{2}}{d r^{2}}+k^{2}\right) \Re_{l}=\bar{V}_{l} \Re_{l}+\int_{0}^{\infty} \bar{U}_{l}\left(r, r^{\prime}\right) \Re_{l}\left(r^{\prime}\right) d r^{\prime} .
$$

This equation in turn leads to the integral equation

$$
\Re_{l}(r)=\Phi_{l}(r)+\int_{0}^{\infty} G\left(r, r^{\prime}\right) \bar{V}_{l}\left(r^{\prime}\right) \Re_{l}\left(r^{\prime}\right) d r^{\prime}+\int_{0}^{\infty} k_{l}\left(r, r^{\prime}\right) \Re_{l}\left(r^{\prime}\right) d r^{\prime},
$$

where $G\left(r, r^{\prime}\right)$ is the Green function corresponding to the inverse of the operator $\left(d^{2} / d r^{2}+k^{2}\right)$,

$$
\begin{aligned}
& G\left(r, r^{\prime}\right)=-\frac{1}{k} \sin (k r) \cos \left(k r^{\prime}\right), \quad r<r^{\prime}, \\
& G\left(r, r^{\prime}\right)=-\frac{1}{k} \cos (k r) \sin \left(k r^{\prime}\right), \quad r^{\prime}<r .
\end{aligned}
$$

In the above $k$ is the asymptotic wave number,

$$
k=\left(\frac{2 m}{\hbar^{2}} E\right)^{1 / 2}, \quad \bar{V}=\frac{2 m}{\hbar^{2}} V, \quad \bar{U}=\frac{2 m}{\hbar^{2}} U,
$$

the kernel $k_{l}\left(r, r^{\prime}\right)$ is given by

$$
k_{l}\left(r, r^{\prime}\right)=\int_{0}^{\infty} G\left(r, r^{\prime \prime}\right) \bar{U}_{l}\left(r^{\prime \prime}, r^{\prime}\right) d r^{\prime \prime}
$$

and $\Phi_{l}(r)$ is a documented function, such as a Ricatti-Bessel function $k r J_{l}(k r)$, for example.

The solution of the equation (35) in the absence of the nonlocal term $k_{l}$ has been described in the atomic-physics literature, see, for example, [28] and references therein. A new accurate and economical spectral solution has been given in [13. The emphasis in the present paper is to investigate under which conditions the solution of (35) in terms of the NCC quadrature is feasible. Two physical examples will be discussed in what follows.

9.2.1. The Perey-Buck example. The nonlocal Perey-Buck [22] potential is of the form

$$
U_{P B}\left(r, r^{\prime}, x\right)=N_{P B}(\rho) V_{P B}(R)=\frac{1}{\left(\pi^{1 / 2} \beta\right)^{3}} e^{-\rho^{2} / \beta^{2}} \cdot \frac{V_{0}}{1+e^{\left(R-R_{0}\right) / \alpha}},
$$

where $\beta, V_{0}, R_{0}$ and $\alpha$ are given constants, and where $\rho=\left|\vec{r}-\vec{r}^{\prime}\right|$ and $2 R=\left|\vec{r}+\vec{r}^{\prime}\right|$. In conventional applications of the Perey-Buck potential the variable $R$ is replaced with $\left(r+r^{\prime}\right) / 2$. In this case the factor $V_{P B}(R)$ can be taken outside the integral in (31), with the result, for $l=0$,

$$
U_{0}\left(r, r^{\prime}\right)=2 \pi\left(\frac{1}{\sqrt{\pi} \beta}\right)^{3} \frac{1}{2} \beta^{2}\left[e^{-\left(r-r^{\prime}\right)^{2} / \beta^{2}}-e^{-\left(r+r^{\prime}\right)^{2} / \beta^{2}}\right] \cdot \frac{V_{0}}{1+e^{\left(R-R_{0}\right) / \alpha}} .
$$


In this case the integration kernel $k_{0}\left(r, r^{\prime}\right)$ is the same whether $r$ is greater or less than $r^{\prime}$, and the NCC quadrature described in this paper is unnecessary.

Even if the variable $R$ were kept in its original form $2 R=|\vec{r}+\vec{r}|$, the resulting expression for $U_{0}\left(r, r^{\prime}\right)$ would still depend only weakly on $\left|\vec{r}-\vec{r}^{\prime}\right|$ in situations encountered in nuclear physics for which the range of the factor $N_{P B}(\beta \sim 1)$ is much smaller than the range of $V_{P B}(2 \alpha \geq 6)$. This is because the integrand in (31) near the lower limit $x \approx-1$ of the integral, $\exp \left[-\left(r+r^{\prime}\right)^{2} / \beta^{2}\right] V_{P B}\left(\left|r-r^{\prime}\right| / 2 \alpha\right)$, is then much smaller than the value of the integrand near the upper limit, $x \approx 1$, $\exp \left[-\left(r-r^{\prime}\right)^{2} / \beta^{2}\right] V_{P B}\left(\left(r+r^{\prime}\right) / 2 \alpha\right)$, and the overall result depends weakly on $\left|r-r^{\prime}\right|$.

This situation would however be quite different if the factor $N_{P B}(\rho)$ were to depend on $\rho$ exponentially, $\alpha \exp (-\rho / \beta)$, rather than $\alpha \exp \left[-(\rho / \beta)^{2}\right]$. The dominant contribution to the integral (31) would again come from the upper limit $x \approx 1$, where the integrand is $\exp \left[-\left|r-r^{\prime}\right| / \beta\right] V_{P B}\left(\left(r+r^{\prime}\right) / 2 \alpha\right)$, and $U_{0}\left(r, r^{\prime}\right)$ would now depend strongly on whether $r<r^{\prime}$ or $r>r^{\prime}$. In this case the NCC quadrature would be relevant, and for this reason Example 2 was described in Section 6 .

9.2.2. The interaction between two nucleons. The interaction between two nucleons can be expressed in terms of the exchange of mesons following the original ideas of Yukawa, and is now used extensively by many researchers, see, for example, [20] and [9]. The resulting $n-n$ potential is usually given in the momentum space, but can also be converted to configuration space by means of Fourier integrals of the momentum space representation. If certain approximations are made, the resulting potential is local and has the form

$$
V_{n-n}=\delta\left(\vec{r}-\vec{r}^{\prime}\right) Y_{\text {reg }}(r) .
$$

The regularized potential $Y_{\text {reg }}(r)$, [9], is such that

$$
Y_{\text {reg }}(r)_{r \rightarrow 0}=\text { constant }+O\left(r^{2}\right), \quad Y_{\text {reg }}(r)_{r \rightarrow \infty} \propto \frac{1}{r} e^{-\mu r},
$$

where $\mu$ is proportional to the mass of the exchanged meson. In the above result, the factor $\sqrt{m^{2} /\left(E_{q} E_{q^{\prime}}\right)}$ has been replaced by a certain approximation, see [20. If however, the exact factor $\sqrt{m^{2} /\left(E_{q} E_{q^{\prime}}\right)}$ is used, then the corresponding nucleonnucleon potential becomes nonlocal in configuration space, see [20, and is shown in 9 to be of the form

$$
V_{n-n}\left(\vec{r}, \vec{r}^{\prime}\right) \propto N_{n-n}(\rho) Y_{\text {reg }}(R) .
$$

In the above $\rho$ and $R$ have the same meaning as in the Perey-Buck example, $V_{\text {reg }}$ is given by the expression (38) with $r$ replaced by $R$, and

$$
N_{n-n}(\rho)=\frac{m^{2}}{2 \pi^{2}} \frac{1}{\rho} K_{1}(m \rho),
$$

where $K_{1}(z)$ is a modified Bessel function defined in reference [1] in Section 9.6. For small values of $z$ we have $K_{1}(z) \approx(1 / z)\left(1+O\left(z^{2}\right)\right)$, and hence, when $\rho \rightarrow 0$,

$$
N_{n-n}(\rho) \approx \frac{m^{2}}{2 \pi^{2}} \rho^{-2},
$$

while for large values of $z$ an asymptotic expansion of $K_{1}$ gives for $N_{n-n}$ the approximation

$$
N_{n-n} \approx \frac{m^{2}}{2 \pi^{2}}\left(\frac{\pi}{2 m}\right)^{1 / 2} \rho^{-3 / 2} e^{-m \rho} .
$$


For the purpose of the present study, we will approximate $V_{n-n}\left(r, r^{\prime}, x\right)$ by

$$
V_{n-n}\left(r, r^{\prime}, x\right) \approx \frac{1}{\rho^{2}} e^{-m \rho} e^{-\mu R}
$$

since this expression captures the nature of the singularity at $\rho \rightarrow 0$, and the nature of the exponential decay at large values of $\rho$ or $R$.

The value of $m(940 / 200 \approx 5)$ is larger than the value of $\mu \approx 1.5$, and hence, similarly to the Perey-Buck case, the factor $N(\rho)$ decays faster than $Y_{\text {reg }}(R)$. Thus, the discussion in the previous subsection on the Perey-Buck example shows that $U_{0}\left(r, r^{\prime}\right)$ should depend strongly on $\left|r-r^{\prime}\right|$, as is indeed the case, as will be shown below. The singularity $\rho^{-2}$ introduces a logarithmic singularity $\ln \left|r-r^{\prime}\right|$ which completely dominates the overall kernel $k\left(r, r^{\prime}\right)$, as will also be described.

The $l=0\left(P_{0} \equiv 1\right)$ value of $V_{n-n}$ is obtained from (7) by considering the contributions to the integral

$$
\int_{-1}^{1} V_{n-n}\left(r, r^{\prime}, x\right) d x
$$

near the lower and upper endpoints, $x=-1$ and $x=1$ respectively, and then taking the sum of the two. Accordingly, an approximation to $\left(U_{n-n}\right)_{l=0}$, with $V_{n-n}$ given in (40), is

$$
\begin{gathered}
\left(U_{n-n}\right)_{0} \approx 2 \pi \ln \frac{\bar{r}}{\left|r-r^{\prime}\right|} e^{-m\left|r-r^{\prime}\right|} e^{-\mu\left(r+r^{\prime}\right) / 2} \\
+2 \pi \ln \frac{r+r^{\prime}}{\bar{r}} e^{-m\left(r+r^{\prime}\right)} e^{-\mu\left|r-r^{\prime}\right| / 2}
\end{gathered}
$$

where $\bar{r}$ is the half-way point between $\left|r-r^{\prime}\right|$ and $r+r^{\prime}$. Its value is $\bar{r}=r^{\prime}$ for $r<r^{\prime}$ and $\bar{r}=r$ for $r^{\prime}<r$. Comparisons of the numerical evaluations of (31) using the expression in (40) for $V_{n-n}$, with the approximate expression (41), showed that the two quantities were equal within a factor of two; in particular, the singularity due to $\ln \left|r-r^{\prime}\right|$ was well represented.

When according to equation (36) $\left(U_{n-n}\right)_{0}$ is convoluted with the Green function so as to obtain the final kernel in the integral equation, the logarithmic singularity is propagated into this final kernel. This occurs when the integration variable $r^{\prime \prime}$ sweeps over the region which surrounds $r^{\prime}$. One finds that when both $r$ and $r^{\prime}$ are close to zero, the singularity of $k\left(r, r^{\prime}\right)$ is proportional to

$$
k\left(r, r^{\prime}\right) \propto\left(r-r^{\prime}\right)^{2} \ln \left|r-r^{\prime}\right|,
$$

and when both $r$ and $r^{\prime}$ are not close to the origin, the singularity is

$$
k\left(r, r^{\prime}\right) \propto\left|r-r^{\prime}\right| \ln \left|r-r^{\prime}\right| .
$$

The presence of this singular behavior affects the spectral accuracy of the NCC quadrature. In the numerical calculations of the accuracy achieved for such singular kernels, presented in the text, the following form of $k$ was assumed based on the discussion above:
a) $k\left(r, r^{\prime}\right)=\left(r-r^{\prime}\right)^{2} \ln \left(r-r^{\prime}\right)^{2} \cdot e^{-\left|r-r^{\prime}\right|} e^{-\left(r+r^{\prime}\right)}$;
b) $k\left(r, r^{\prime}\right)=\left|r-r^{\prime}\right| \ln \left(r-r^{\prime}\right)^{2} \cdot e^{-\left|r-r^{\prime}\right|} e^{-\left(r+r^{\prime}\right)}$. 


\section{REFERENCES}

1. M. Abramovitz and I. Stegun (Eds.), Handbook of Mathematical Functions, Dover, NY, 1972. MR 94b:00012

2. B.K. Alpert, Hybrid Gauss-Trapezoidal Quadrature Rules, SIAM J. Sci. Comput. 20, 5 (1999), pp. 1551-1584. MR 2000m:41044

3. P.M. Anselone, Collectively Compact Operator Approximation Theory and Applications to Integral Equations, Prentice-Hall, Englewood Hills, 1971. MR 56:1753

4. K.E. Atkinson, A Survey of Numerical Methods for the Solution of Fredholm Integral Equations of the Second Kind, SIAM, Philadelphia, 1976. MR 58:3577]

5. C.T.H. Baker, The Numerical Treatment of Integral Equations, Oxford University Press, 1977. MR 57:7079

6. R.H. Chan and M.K. Ng, Conjugate Gradient Methods for Toeplitz Systems, SIAM Review 38, 3 (1996), pp. 427-482. MR 97i:65048

7. C.W. Clenshaw and A. R. Curtis, A method for numerical integration on an automatic computer, Numer. Math. 2 (1960), pp. 197-205. MR 22:8659

8. L.M. Delves and J.L. Mohamed, Computational Methods for Integral Equations, Cambridge University Press, Cambridge, 1985. MR 87j:65159

9. Ch. Elster, E.E. Evans, H. Kamada and W. Gloeckle, Nonlocality in the Nucleon-Nucleon Interaction Due to the Minimal-Relativity Factors: Effects on Two-Nucleon Observables and the Three-Nucleon Binding Energy, Few-Body Systems, 21, 25 (1996), pp. 25-45.

10. H. Feshbach, A Unified Theory of Nuclear Reactions II, Ann. Phys. NY, 19 (1962), pp. 287-313. MR 25:4863

11. I. Gohberg and I.A. Fel'dman, Convolution Equations and Projection Methods for Their Solution, Transl. Math. Monograph, Vol 41, American Mathematical Society, Providence, RI, 1974. MR 50:8149

12. I. Gohberg, S. Goldberg and M.A. Kaashoek, Classes of Linear Operators, Vol. 1, Birkhauser Verlag, Basel, 1990. MR 93d:47002

13. R.A Gonzales, J. Eisert, I. Koltracht, M. Neumann and G. Rawitscher, Integral Equation Method for the Continuous Spectrum Radial Schrödinger Equation, J. of Comput. Phys. 134 (1997), 134-149. MR 98i:81040

14. D. Gottlieb and S. Orszag, Numerical Analysis of Spectral Methods, SIAM, Philadelphia, 1977. MR 58:24983

15. L. Greengard and V. Rokhlin, On the Numerical Solution of Two-Point Boundary Value Problems, Commun. Pure Appl. Math., 44 (1991), pp. 419-452. MR 92a:34018

16. S.-Y. Kang, Numerical Solution of Integral Equations with Nonsmooth Kernels and Applications, Ph.D. Thesis, Department of Mathematics, University of Connecticut, Storrs, CT, 2000 .

17. S. Kapur and V. Rokhlin, High Order Corrected Trapezoidal Quadrature Rules for Singular Functions, SIAM J. Numer. Anal., 34, 4 (1997), pp. 1331-1356. MR 98k:65011

18. R.H. Landau, Quantum Mechanics II, John Wiley, New York, 1990. MR 91g:81001

19. R. Machleidt, K. Holinde and Ch. Elster, The Bonn Meson-Exchange Model for the NucleonNucleon Interaction, Phys. Rep. 149, 1 (1987), pp. 1-89.

20. R. Machleidt, F. Sammarruca and Y. Song, Nonlocal Nature of the Nuclear Force and its Impact on Nuclear Structure, Phys. Rev. C 53, R1483 (1996), pp. 1483-1487.

21. N.F. Mott and H.S. Massey, The Theory of Atomic Collision, 3rd ed. Oxford at Clarendon Press, 1965.

22. F. Perey and B. Buck, A Nonlocal Potential Model for the Scattering of Neutrons by Nuclei, Nucl. Phys. 32, 353 (1962), pp. 353-380.

23. G.H. Rawitscher, B.D. Esry, E. Tiesinga, P. Burke, Jr. and I. Koltracht, Comparison of Numerical Methods for the Calculation of Cold Atomic Collisions, J. Chem. Phys. 111, 23 (1999), 10418-10426.

24. G.H. Rawitscher, S-Y. Kang, I. Koltracht, E. Zerrad, K. Zerrad, B.T. Kim and T. Udagawa, Comparison of Numerical Methods for the Solution of the Schrödinger Equation in the Presence of Exchange Terms, submitted.

25. L. Reichel, Fast Solution Methods for Fredholm Integral Equations of the Second Kind, $N u$ mer. Math. 57 (1989), pp. 719-736. MR 91i:65211

26. H.L. Royden, Real Analysis, 3rd Edition, Macmillan Publishing Company, NY, 1988. MR 90g:00004 
27. W.N. Sams and D.J. Kouri, Noniterative Solutions of Integral Equations for Scattering. I. Single Channels, J. Chem. Phys. 51 (1969), pp. 4809-4814. MR 58:7006

28. E.R. Smith and R.J.W. Henry, Noniterative Integral-Equation Approach to Scattering Problems, Phys. Rev. A 7, (1973), pp. 1585-1590. MR 50:15691

29. J. Strain, Locally Convergent Multidimensional Quadrature Rules for Singular Functions, SIAM J. Sci. Comput. 16, 4 (1995), pp. 992-1017. MR 96b:65026

Department of Mathematics, Purdue University North Central, Westville, Indiana 46391

E-mail address: skang@purduenc.edu

Department of Mathematics, University of Connecticut, Storrs, Connecticut 06269

E-mail address: kolt@math.uconn.edu

Department of Physics, University of Connecticut, Storrs, Connecticut 06269

E-mail address: rawitsch@uconnvm.uconn.edu 